\title{
Réinsérer la Serbie dans l’analyse de la transition. RAPPORTS DE PROPRIÉTÉ, ÉTAT ET SALARIAT
}

\author{
Catherine SAMARY *
}

\author{
Revue d'Etudes comparatives Est/Ouest \\ Volume 35 - mars-juin $2004-\mathrm{n}^{\circ} 1-2$ \\ CNRS
}

\author{
Mis en ligne sur http://semimarx.free.fr/article.php3?id_article $=190$ \\ et http://www.europe-solidaire.org/spip.php?article7526
}

Résumé : La Serbie dirigée par Slobodan Milosevic a été présentée comme le dernier des pays socialistes européens à résister aux privatisations. La transition y aurait débuté après la "révolution" démocratique du 5 octobre 2000. Une telle approche occulte des caractéristiques générales de la transition, dans ses diverses phases. Les nouvelles lois serbes de privatisation marquent, à nos yeux, non pas le début de la transition (qui commença dans le cadre yougoslave en 1989) mais une accentuation radicale de la logique libérale s'inscrivant dans des tendances plus générales. Réinsérer la Serbie dans l'analyse comparative de la transition permet d'expliciter les critères, enjeux et contradictions de cette dernière. Nous le ferons en nous centrant sur les dimensions socio-économiques essentielles des transformations en cours : les rapports de propriété au cœur des systèmes qui sont tributaires de la place des relations marchandes et de la logique socio-économique de l'État. Notre approche se voudrait une contribution aux analyses comparatives des systèmes contemporains et des transitions. Nous tenterons d'expliciter en quoi le cas de la Yougoslavie titiste éclaire l'aspect général de droits de propriété (ou de relations principal/agent) "impurs" dans les pays socialistes pris dans leur ensemble et en quoi les conflits provoqués par la transition en Serbie rejoignent ceux que cèlent les changements de système sans "révolution".

Abstract: Slobodan Milosevic's Serbia was often deemed as the last European former Socialist country holding out against privatisation. The transition supposedly started there with the democratic "revolution" of 5 October 2000. This view keeps us from seeing the overall characteristics of the transition during its various phases. In fact, Serbia's new laws on privatization did not mark the start of the transition (which actually began in 1989 in Yugoslavia); instead they signaled a radical shift towards liberalism, in line with more general trends. By reinstating Serbia in a comparative analysis of transitions, light is shed on the criteria, issues and contradictions of these transitions. We will focus on the essential socio-economic dimensions of the ongoing transformations : the property relationships, at the very heart of systems. They depend on the place of market relationships and of the socio-

\footnotetext{
* Maître de conférences à Paris-Dauphine, chercheur à l'IRISES-Dauphine/CNRS (Institut de recherches interdisciplinaires en sociologie, économie, et sciences politiques Université Paris Dauphine, place du Maréchal de Lattre de Tassigny, 75016 Paris ; catherine.samary@dauphine.fr).
} 
economic logic of the state. This attempt to contribute to the comparative analysis of transitions in contemporary systems focuses on Yugoslavia under Tito in order to shed light on the general characteristic of "impure" property rights (or principal/agent relations) in Socialist countries. We thus see why conflicts during the Serbian transition highlight those, more hidden, behind the changes in systems that have not undergone a "revolution".

\section{INTRODUCTION}

La Serbie dirigée par Slobodan Milosevic a été présentée comme le dernier des pays socialistes ${ }^{1}$ européens à résister aux privatisations : la transition y aurait débuté après la "révolution" démocratique du 5 octobre 2000. Nous contestons cette approche qui occulte des caractéristiques générales de la transition dans ses diverses phases, les toutes dernières lois serbes sur la privatisation s'inscrivant, à nos yeux, dans une accentuation radicale de la logique libérale à la fin des années 1990. Réinsérer la Serbie dans l'analyse de la transition permet en fait d'expliciter les enjeux de celle-ci.

La notion de transition s'est imposée après la chute du Mur de Berlin pour désigner le basculement des pays socialistes vers l'économie de marché telle que la conçoivent les institutions de la mondialisation ${ }^{2}$. Elle exprimait le fait que des éléments de rupture intervenaient, remettant en cause des traits essentiels de l'ancien système et se distinguant en cela des réformes du passé. Mais comment se déterminait, dans les sociétés en transition, le sens (signification et direction) des changements?

Alors que la chute du parti unique était initialement considérée comme le signe caractéristique du début de la transition, le cas de la Chine élargissait le débat sur les scénarios possibles et infléchissait les critères retenus vers les dimensions socio-économiques (RECEO, 1999a) sans que le

1 La notion de "pays socialiste" doit être entendue ici comme un raccourci descriptif pour désigner les pays se réclamant du projet socialiste. On sait que le discours apologétique des régimes concernés dissimulait sous cette étiquette les écarts entre la réalité et les buts proclamés (émancipation des travailleurs, satisfaction des besoins). Nous chercherons à montrer que ces écarts ont été à la source de contradictions significatives, voire de la crise générale de ces systèmes.

2 Voir les publications des institutions internationales (Banque Mondiale, OCDE, FMI), de l'UE (notamment, le Centre for Economic Policy Research) ainsi que le site et les publications du Département des relations économiques extérieures - DREE - du ministère français des Finances. 
passage des réformes de l'ancien système à un changement de la logique d'ensemble soit toujours clair ${ }^{3}$. Et si la Chine, qui reste une dictature gouvernée par un parti unique, était en transition, pourquoi le régime autoritaire de S. Milosevic, doté d'un pluralisme politique, fut-il de façade, ne l'aurait-il pas été ?

La difficulté de déterminer les points de rupture significatifs se combine avec I'héritage et l'actualisation d'anciens débats conceptuels sur le " socialisme réel " ${ }^{4}$. Certains chercheurs ont mis la Yougoslavie titiste "à part" (Chavance, 1994, 1996, 1999; Motamed-Nejad, 1999) en raison de ses réformes des années 1960-1970 (elles ont notamment donné un rôle distinctif au marché) qui l'ont éloignée de ce qu'ils considèrent être la norme de l'ancien système socialiste. Nous estimons, au contraire, que le socialisme autogestionnaire yougoslave éclaire les contradictions générales de ce système.

Comprendre, au-delà des différences, ce qui relève d'une même matrice de problèmes et de concepts représente l'une des difficultés des analyses comparatives mais fait aussi leur intérêt, particulièrement lorsqu'elles portent sur le tournant historique que fut la fin de I'URSS. L'expérience de plus de dix ans de transition, qui a fait l'objet de bilans issus d'horizons divers ${ }^{5}$, permet de tester les critères retenus; elle donne du recul pour apprécier les traits rapprochant de manière significative des cas relevant de la même "espèce" de système (Kornai, 1990, 1996, 2001 ; Chavance, Magnin, Motamed-Nejad \& Sapir, 1999). La discussion concernera ici les noyaux durs communs à des formations sociales historiques

3 La Chine, le Vietnam et Cuba, tous trois dirigés par des partis communistes, doivent-ils pour autant être caractérisés de la même façon sur le plan conceptuel ? Telle est notamment la thèse défendue par l'ouvrage Le socialisme de marché à la croisée des chemins (ANDRÉANI, 2003).

4 Pour des retours sur ces questions, lire en particulier Andreff, 1993 ; Chavance, Magnin, Motamed-Nejad \& SAPIR, 1999 ; Mandel, 1991 ; Motamed-Nejad, 1997 ; Roland, 1989 ; SAMARY, 1999d.

5 On peut se référer en la matière à RECEO, 1999a et b; Nouveaux cahiers de l'IUED, 2001 ; les Études du CERI, notamment pour la période 1999-2001; le Courrier des pays de l'Est des années 1999-2001; les Études du CEDUCEE et de la Documentation française, en particulier LHOMEL, 2001. Voir également ANDREFF, 2002 ; CLÉMENT, 1999 ; Gorzelak, Ehrlich, Faltan \& ILlner, 2001 ; IRM, 1996 ; Kornai, 2001 ; Mink \& SzUrek, 1999 ; Menciger, 2000 ; Motamed-NeJad, 1999; NAGels, 1991, 1996 ; PaPIC, 2001 ; SAmary, 1996, 1999b; Sapir, 1999 ; Solioz \& Dizdarevic, 2001, 2003. 
diverses, dites socialistes ${ }^{6}$, qui permettent de penser qu'elles appartiennent effectivement au même système. Dans cette diversité, pèsent les résistances d'un certain passé - il n'est pas le même pour tous - ou des luttes préfigurant d'autres possibles qui laisseront des traces dans les réformes. La Yougoslavie titiste en fut un creuset fort riche mais nous ne développerons pas la genèse et les caractéristiques de son "modèle", qui s'était très tôt différencié de celui de l'URSS, puis de sa crise ${ }^{7}$. Nous nous concentrerons sur les points communs et les enjeux structurels socio-économiques rapprochant I'URSS, I'Europe de l'Est et la Yougoslavie titiste, les crises qu'elles ont traversées et la transition vers le capitalisme des années récentes. C'est dans les rapports de propriété que nous tenterons de cerner ces enjeux.

Cette notion, empruntée au marxisme, recouvre les relations sociales de production et de distribution, lesquelles sont tributaires de la place des rapports marchands et des logiques sociales défendues par l'État. Nous entendons analyser la transformation de ces relations sociales qui affecte les droits de propriété juridiques et réels (droits d'utiliser un actif, d'en tirer un revenu et de céder cet actif de manière définitive à un tiers). L'analyse marxiste des conditions d'émergence puis de domination de rapports sociaux capitalistes ${ }^{8}$ - avec la libération de la force de travail, la privatisation des moyens de production et l'extension des relations monétaires - nous semble éclairante appliquée à un contexte historique non prévu par Marx: la transition au capitalisme mondialisé de pays ayant voulu instaurer un tout autre système.

6 Soulignons que pour les marxistes soviétiques des années 1920 (plus particulièrement, N. I. Boukharine et E. Preobrajenski), la désignation de l'URSS comme " socialiste " indiquait non pas une réalité mais un objectif. Pour eux, la société n'était plus dominée par la logique capitaliste tout en n'ayant pas encore véritablement accédé au socialisme. Les débats conceptuels adhérant à ces thèses, qui demeurent pour nous très éclairants, se heurtaient au fait que les catégories économiques en usage dans les sociétés marchandes capitalistes (prix, profit, salaire...) s'avéraient à la fois nécessaires et insatisfaisantes pour analyser la nouvelle société. À ce sujet, cf. notamment W. Brus (1968), E. Mandel (1974) et B. Bettelheim (1968). 7 Nous ne pouvons notamment pas traiter ici de la question très controversée des facteurs internes et externes de l'éclatement de la Yougoslavie. Cf., dans des approches différentes, Johnstone, 2002 ; Magas, 1993 ; Morokvasic, 1992; Samary, 1994, 2000 ; Stark, 1993 ; WOODWARD, 1995.

8 Sur l'émergence du capitalisme, lire MARX (a), pp. 1167-1237 ou MarX (b) pp. 312-355. 
L'approche libérale des droits de propriété 9 rejoint paradoxalement le marxisme en ce qu'elle met, elle aussi, à sa façon, l'accent sur les relations sociales que cachent les rapports aux choses. Nous utiliserons en partie sa problématique sans adhérer à ses présupposés et conclusions normatives (la préconisation des privatisations généralisées, réputées être toujours plus efficaces). Nous soutiendrons l'idée que le caractère hybride ou non transparent des rapports (droits) de propriété supposés collectifs et du rapport principal/agent est significatif de l'ancien système et de sa crise.

Nous survolerons ici la question des moyens (institutionnels, politiques, socio-économiques) convenant à des droits de propriété (d'appropriation) partagés. La suprématie d'un parti unique régnant au nom des travailleurs (du peuple ou de la société) fut une forme de confiscation de ces droits, ce qui a nourri les thèses d'un parti-classe (ou parti-État) propriétaire réel des moyens de production. Toutefois, ces thèses ne prennent pas en compte l'autre facette d'une même réalité : l'abolition de la propriété privée des moyens de production et du marché et la perte de leur caractère dominant au profit d'une propriété sociale que les pouvoirs en place étaient uniquement censés gérer. La réalité limitée de leurs droits de propriété faisaient d'eux plutôt les agents d'une relation principal/ agent où le principal était la société ou l'ensemble des travailleurs. L'absence d'un pouvoir réel de contrôle organisé va marquer ce que nous appelons l'aspect hybride de ces rapports de propriété. Mais la voie choisie n'éliminera pas une véritable contradiction : d'un côté, l'instauration d'une propriété collective et, de l'autre, le monopole de sa gestion par le parti. Ce double aspect permet d'interpréter les crises ayant éclaté quand les travailleurs/propriétaires théoriques ont exigé la réduction de l'écart entre les la doctrine et la réalité comme, par exemple, en Pologne et en Hongrie en 1956 et, de nouveau, en Pologne des années plus tard sous l'impulsion de Solidarnosc. Mais il éclaire également nombre des réformes introduites par le parti unique dans le but de contenir ces tensions pour prolonger son règne. Elles prennent souvent la forme d'une répression des débordements dangereux pour le monopole du pouvoir du parti-État couplée à la reconnaissance de certains droits de propriété sociale, constituant une autre illustration de la contradiction du système. Dans tous ces domaines, le cas yougoslave indique mieux que tout autre la nature contradictoire de ces régimes et ne s'en distingue nullement. C'est pourquoi, il ne serait pas pertinent de l'en dissocier.

Dans les limites de cet article, nous ne pouvons analyser l'ensemble des

9 Nous adhérons largement aux idées développées par B. Coriat et O. Weinstein (1995) et au jugement qu'ils portent sur les nouvelles théories de l'entreprise et, plus spécialement, la théorie néo-classique des droits de propriété. 
institutions concernées par les rapports de propriété de l'ancien et du nouveau système en transition. Nous mettrons l'accent sur l'État et sa redéfinition sociale et territoriale conjuguée avec l'objectif d'appropriation privée des richesses (en nous appuyant sur le cas yougoslave). Derrière le changement de logique de l'État, nous retrouvons la "grande transformation" capitaliste (Polanyi, 1957) portant sur les rapports de propriété émergents et, tout d'abord, la transformation de la monnaie en capital. Nous entendons celui-ci au sens marxiste de capital-argent susceptible d'être accumulé et de se métamorphoser en capital-productif et capital-marchand en quête de profit monétaire dont l'appropriation se fonde sur la propriété privée des moyens de production. Cette transformation devrait, pour aller à son terme dans une société industrielle, s'accompagner de l'affranchissement de la force de travail de toute forme de protection sociale non marchande et de l'émergence d'un vrai salariat. Aussi notre analyse rejoint-elle celle de J. Nagels $(1991,1996)$ pour qui la transition tend vers le "capitalisme sauvage". Mais nous insisterons sur les difficultés symptomatiques de la privatisation des grandes entreprises où se nouaient, justement, les anciens rapports de propriété dont nous soulignons le caractère impur.

Nous procéderons en trois temps en analysant d'abord les caractéristiques de la transition en général (1) avant de remonter au noyau dur de l'ancien système socialiste où s'incorpore le cas yougoslave (2) et de rencenser les étapes de la transition serbe, initialement yougoslave, comparée à celle de la Slovénie jusqu'au récent tournant (3). Celui-ci éclaire les enjeux de la transition en général et, sans doute, la fragilité d'une construction européenne où le libéralisme tend à l'emporter.

\section{De la transition EN GÉNÉRAL}

\subsection{Du rejet du parti unique auX “ DÉMOCRatures ”}

En rejetant le système dictatorial de l'ancien parti-État unique, la grande masse des populations, et notamment lors du congrès de Solidarnosc en Pologne en 1980, ne s'est jamais mobilisée pour un projet de privatisation généralisée. Le but était de vivre mieux et plus libre en dehors de tout débat sur des " ismes" ou des idéologies brouillées, l'espoir étant souvent de bénéficier de ce que chaque système avait de meilleur (Samary, 1992).

Ce rejet facilita, comme en Pologne ou en Tchécoslovaquie, la formation de larges coalitions lors des premières élections libres. Mais ces fronts "contre" se 
sont ensuite partout divisés au cours de la décennie 1990 dès qu'il s'est agi de définir des projets concrets. Les courants libéraux prétendaient remettre en question l'arbitraire et les gaspillages de l'ancien système mais, aussi, la protection sociale. C'est en partie pourquoi les résultats électoraux ont varié au gré des promesses des nouveaux et anciens partis plus ou moins réformés et de ce que telle ou telle fraction de la population (en position plus ou moins bonne pour affronter l'incertitude du marché) voulait prioritairement exprimer (Lazic, 1995) : une sanction contre les anciens dirigeants corrompus, une volonté de changement radical ou encore la peur de changements perçus, à tort ou à raison, comme menaçants. Slobodan Milosevic joua de tous ces facteurs à la fois.

Dès 1990, le pluralisme politique était instauré dans l'ensemble des républiques yougoslaves comme dans les autres pays d'Europe centrale et orientale (PECO) ${ }^{10}$. Dans plusieurs pays (en Roumanie ou en Slovaquie au début des années 1990 et non pas uniquement en Serbie), les citoyens ont voté, dès les premières élections libres, pour les partis incarnant le mieux une continuité avec le passé de crainte d'une régression ou d'un abandon de la protection sociale.

L'alternance politique indiqua la consolidation d'un certain État de droit. Mais dans les pays-clés de la transition, à commencer par la Pologne, les premières coalitions ayant mené des politiques libérales furent rapidement battues aux élections par les anciens communistes, souvent reconvertis sous l'étiquette socialiste (Drweski, 2002), également adoptée dès le début de la décennie 1990 par le nouveau parti de Slobodan Milosevic. Là où les coalitions anti-communistes avaient d'abord été portées au pouvoir, elles se sont disloquées et effondrées alors que progressaient dangereusement l'abstentionnisme et les votes au profit de la droite xénophobe (Rupnik, 2001).

On constate donc une absence de soutien et de mobilisation populaires en faveur des changements. Celle-ci est paradoxale si l'on se réfère au thème de la démocratisation propre à la transition, supposée promouvoir une plus grande efficacité. Les néologismes sont dès lors fort utiles pour décrire la transition : derrière les désillusions et la désaffection populaires, n'y avait-il pas des choix échappant au débat en dépit de parlements élus? Des variantes de " démocratures" "11 au plan politique ? Et n'avait-on pas assisté, à un niveau plus

10 Pour nous cantonner à la Serbie, sous le régime de Milosevic, les partis de l'opposition, après plusieurs échecs, remportèrent les élections municipales de 1996 dans les principales villes, dont Belgrade.

11 Combinaison de démocratie et de dictature, selon la formule importée d'Amérique latine et reprise par l'écrivain croate Predrag Matvejevitch (1996) pour décrire notamment la Croatie de Tudjman. Celle-ci, en fait, avait beaucoup de traits communs avec la Serbie 
structurel, à des "réfolutions" (néologisme créé par T. Garton Ash [1993] et repris par Chavance, 1999), en partie révolutions au sens d'un changement radical de système - mais sans réelles innovations historiques - et en partie réformes, celles introduites par les appareils en place ? Aussi faut-il expliciter les causes de ce qui s'avère être des traits généraux de la transition.

Initialement, la force des nouveaux courants libéraux était de prétendre apporter à la fois efficacité économique et libertés sur la base des préceptes se disant universels du consensus de Washington. Pour l'immense majorité des gens, marché et privatisation étaient des abstractions que les économistes, souvent moins discrédités que les partis politiques, étaient censés maîtriser. Prétendant à la scientificité, les préceptes néo-libéraux avaient un caractère volontariste et normatif comme le souligne R. Motamed-Nejad (1999, p. 15) : ils se sont imposés en excluant leurs choix du domaine des débats démocratiques (Sapir, 2002). Ils bénéficiaient pour ce faire, non seulement du patronage des institutions de la mondialisation, mais du soutien zélé d'anciens membres de la nomenklatura.

Paradoxalement, en effet, cette aspect non démocratique du consensus de Washington facilita le basculement socio-politique et idéologique de bon nombre d'anciens dirigeants du parti unique vers les privatisations, à des rythmes et sous des étiquettes variables. Tout en se réclamant des nouveaux dogmes, ils s'efforcèrent de transformer les privilèges liés à leurs anciennes fonctions en privilèges de la propriété (Eyal, Szelenyi \& Townsley, 1998 ; Mink \& Szurek, 1999; Szalai, 1999; Drweski, 2001). C'est précisément dans l'analyse des formes prises par les privatisations que l'on peut trouver le pourquoi et le comment de ces "réfolutions" ambiguës.

\subsection{QUELLES PRIVATISATIONS COMME NOYAU DUR DE LA TRANSITION?}

Les privatisations ont été placées au centre de la transition. Mais il faut établir une distinction majeure entre la " petite privatisation " (correspondant principalement à la création de nouvelles et petites entreprises) et la " grande privatisation " (concernant les grandes entreprises, c'est-à-dire aussi l'essentiel de l'emploi et de la production de ces pays industrialisés) ${ }^{12}$.

de Milosevic (GlamocaK, 2002 ; Masson, 2002).

12 Étant donné le poids de son agriculture (environ $25 \%$ du PIB et près de $80 \%$ de la population active dans les années 1990 encore), la Chine se différencie grandement de l'URSS (moins de $10 \%$ du PIB et quelque $12 \%$ de la population active) et des PECO. Dans les dix pays ayant adhéré à l'UE en 2004, l'agriculture occupe en moyenne $21 \%$ de la population active (un peu moins de $40 \%$ en Roumanie) et représente moins de $10 \%$ du PIB. 


\subsubsection{La petite privatisation}

Cette dernière a généralement été le moteur de la croissance des PECO, notamment en Pologne (Duchêne \& Rusin, 2002). On lui a parfois accordé une valeur excessive, divers auteurs, qui s'inspiraient de l'école autrichienne libérale de Hayek ou encore du courant "évolutionniste" ${ }^{13}$, y ayant vu la voie privilégiée de la transition. Il s'agissait en tout cas de créer à la fois un mécanisme concurrentiel, de vrais propriétaires et un transfert plus ou moins rapide, selon les approches, des financements vers les nouvelles firmes privées (start-up). Les exemptions d'impôt initiales accordées aux nouvelles entreprises ont généralement favorisé ce processus.

Toutefois, ces petites entreprises sont souvent fragiles et leur croissance atteint très rapidement des limites. En témoignent non seulement le ralentissement de la croissance polonaise après 1998 mais, surtout, le contenu de cette croissance marquée, dans la décennie 2000, par une nouvelle augmentation du chômage de la population active ${ }^{14}$ dépassant $18 \%$ en moyenne en 2004. Une étude portant sur les petites privatisations dans les pays issus de l'ex-Yougoslavie (hormis la Bosnie-Herzégovine) depuis 1990 indique qu'en dépit de dizaines de milliers de création d'entreprises, " la plupart d'entre elles soit n'ont jamais été opérationnelles, soit ont fait faillite mais sans être rayées du registre du commerce" (Bartlett, 1999).

En outre, en Pologne et en Yougoslavie, la majeure partie des terres appartenait à de petits paysans et toutes les réformes entreprises dans le cadre de l'ancien système ont été des ouvertures à la petite propriété personnelle, artisanale ou paysanne. Une partie substantielle des lopins de terre privés complétait le niveau de vie et les avantages sociaux liés au travail en entreprise sous la forme de l'autoconsommation ou de la vente de produits agricoles, source de revenus. Autrement dit, malgré une idéologie dominante le plus souvent hostile ou méfiante à l'égard de la propriété privée et dans des limites étroites, la petite propriété personnelle avait trouvé sa place et ses appuis dans le système. D'où l'apparent paradoxe d'une petite paysannerie privée, en Pologne comme en Serbie, s'avérant être une clientèle électorale essentielle des partis les plus populistes issus de l'ancien parti unique car se sentant menacée par des partis libéraux favorables aux privatisations... mais sans accompagnement social.

13 Pour une présentation de ces diverses approches de la transition, cf. ANDREFF, 2002.

14 La population active agricole, en augmentation en Pologne comme dans les autres PECO à l'exception de la République tchèque, traduit sans doute un chômage caché. À ce propos, voir les données des études annuelles produites par Le Courrier des pays de l'Est, Documentation française. 
Sous l'angle retenu ici, la petite propriété privée (sans personnel salarié) ne permet pas de caractériser la transition entre systèmes parce qu'on peut la retrouver sous diverses formes dans les différents types de systèmes concernés. Et nulle part, en pratique, les petites privatisations n'ont résolu le problème de la restructuration des grandes entreprises dont le coût social, économique et politique était au centre des difficultés de la transition.

\subsubsection{Les grandes privatisations, enjeux de la transition}

C'est donc bien la privatisation des grandes entreprises qui est significative des enjeux et des difficultés de la transition. Pour les mettre en évidence, nos réflexions porteront sur les scénarios de la toute première période de la transition (celle des "réfolutions ", à distinguer par conséquent des réformes de l'ancien système). D'une part, l'on assiste au basculement de l'État vers une logique socio-économique nouvelle. D'autre part, le critère de différenciation des grandes privatisations fut, fondamentalement, l'apport ou non de capital (seule la première variante correspond à une vente réelle). Enfin, nous interprèterons les privatisations de masse introduisant un actionnariat populaire comme une voie paradoxale de transition vers le salariat.

\subsubsection{Quel capital pour acheter?}

Le choix de la vente était paradoxal du fait de la considérable insuffisance de capital-argent national accumulé pour acheter les entreprises, une insuffisance à nos yeux significative de toute la transition. Elle renvoie, en effet, à l'absence de marché du capital et aux fonctions limitées de la monnaie dans le cadre des rapports de propriété de l'ancien système. L'économiste polonais W. Brus a, dès 1968, analysé ce qu'il appelait le "rôle passif" de la monnaie dans un système sans réelle relation d'achat-vente des moyens de production et où les prix ne correspondaient nullement à ceux d'une économie de marché et ses ajustements ; en 1984, l'économiste hongrois J. Kornai est allé dans le même sens, soulignant la "contrainte budgétaire molle" pesant sur les entreprises. $\mathrm{Au}$ plan socio-économique (intégrant une dimension politique), ces caractéristiques non marchandes interdisaient pratiquement la mise en faillite des entreprises. Même si les réformes ont donné plus de latitude aux mécanismes marchands, l'exemple yougoslave étant le plus extrême à cet égard, la substance des critères de rentabilité marchande capitaliste est restée fondamentalement étrangère à la logique de ces systèmes. Ils assuraient une production organiquement extensive, peu soucieuse des coûts, notamment monétaires, révélant pleinement de ce point de vue une essence non capitaliste. Et c'est pourquoi, dans l'ensemble, 
l'accumulation de capital-argent n'a pu réellement commencer qu'après le début de la transition, souvent sous les formes violentes et " primitives" (Slim, 1996) d'un "capitalisme sauvage" (Nagels, 1991, 1996). C'est aussi la raison pour laquelle le critère d'appropriation (centralisée ou non) "du surplus monétaire des entreprises" avancé par R. Motamed-Nejad (1999, p. 13) pour dégager de façon comparative l'essence d'un système n'est pas, à nos yeux, convaincant. Pour l'être, il faudrait que dans tous les systèmes comparés, les prix, la monnaie et donc aussi le surplus monétaire relèvent d'une logique marchande. Ce qui n'est vrai que dans le capitalisme.

Le fait que la privatisation par vente n'ait, majoritairementl, trouvé comme acheteur que le capital étranger indique à quel point le surplus monétaire était faible dans l'ancien système. L'ouverture au capital étranger et, plus largement, aux investissements directs étrangers (IDE) est souvent présentée comme une obligation (il n'y aurait pas d'autre choix) ou une évidente nécessité ; les réticences à cet égard ne relèveraient que d'un nationalisme étroit. Pourtant, la corrélation bénéfique escomptée entre croissance et IDE est loin d'être confirmée (Menciger, 2002). Et l'on connaît bien le conflit d'interprétation récurrent qu'elle suscite : est-ce l'IDE qui induit la croissance ou la croissance constatée qui attire l'IDE (Andreff, 2001) ? Les IDE obéissent à une logique d'agglomération: ils se dirigent plus particulièrement là où se trouvent les infrastructures les meilleures, c'est-à-dire les capitales et les régions les plus développées ou limitrophes de l'UE, creusant les écarts entre pays et, au sein des pays, entre régions. Leur stabilité, par différence supposée avec les placements de portefeuille, n'est pas non plus avérée. Enfin, l'augmentation espérée des capacités de recherche et développement dans les technologies avancées a été "systématiquement surestimée" comme le souligne un rapport émanant d'experts des pays du groupe de Visegrad (Gorzelak, Ehrlich, Faltan \& Illner, 2001) ; les IDE ont même eu pour effet de "réduire les potentiels de R\&D" (ibid., p. 10) dans un contexte de baisse des dépenses publiques sous la pression des préceptes dominants.

Autrement dit, il y a matière à dilemme et solutions de rechange. La Slovénie a fait preuve, tout au long de la décennie 1990, d'une résistance au libéralisme sur laquelle nous reviendrons. En pratique, seules la Hongrie et l'Estonie ont opté pour des privatisations par vente au capital étranger au début de la transition. L'objectif d'un arrimage rapide à l'Occident pour se dissocier de l'URSS a sans doute pesé sur l'attitude de l'Estonie. Quant aux dirigeants de la Hongrie socialiste, ils avaient retenu cette solution dès les années 1980 afin de rembourser la dette extérieure en devises fortes accumulée dans les années 1970: la vente d'une partie des fleurons de l'industrie visait à limiter la 
politique d'austérité. Également confrontées à une crise de la dette, la Roumanie de Ceaucescu et la Yougoslavie des années 1980 ont connu des scénarios de crise et de transition différents. La première a infligé à son peuple une cure d'austérité et de répression de nature explosive mais qui a permis de rembourser intégralement la dette; la seconde a subi l'hyperinflation, la multiplication des grèves et la paralysie de la Fédération avant son éclatement final.

Les choix spécifiques des dirigeants hongrois et la position géostratégique de leur pays dans l'orbite de l'Union européenne firent de celui-ci le principal pays d'accueil des IDE en Europe de l'Est pendant les premières années de la décennie $1990^{15}$.

\subsubsection{Les " privatisations directes" sans apport de capital}

Les grandes privatisations furent, très majoritairement, réalisées quasiment sans apport de capital. L'économiste polonaise Maria Jarosz (2000, p. 11) qualifie cette procédure de "privatisation directe". Il s'agit d'un changement juridique de la propriété qui devait rendre possible le changement de logique socio-économique et de statut des travailleurs. Mais ce processus s'est déroulé dans l'opacité, d'autant que les autorités cherchaient à le légitimer aux yeux des populations en différant dans l'immédiat le démantèlement du système de protection sociale. C'est pourquoi les privatisations directes ont, partiellement, reflété la volonté initiale de préserver un caractère national, voire populaire, aux privatisations. Cette exigence de légitimation a transformé en avantage le manque de capital national ou étranger disposé à racheter les entreprises offertes. Pourtant, à terme, les privatisations sans apport de capital ont posé un problème majeur pour les restructurations recherchées. La tendance des IDE à se concentrer sur les régions les plus riches, évoquée plus haut, explique que les privatisations sans capital concernent en premier lieu les régions les plus sinistrées. Celles-ci sont souvent localisées autour des grandes entreprises de l'ancien système, les plus coûteuses (socialement et économiquement) à restructurer pour des raisons systémiques sur lesquelles nous reviendrons.

C'est dire que les bilans euphoriques et agrégés des privatisations sont trompeurs. Ils recouvrent, pour l'essentiel, deux grandes variantes de privatisation directe, souvent laissées au choix des travailleurs dans la plupart des pays concernés au début de la transition : des privatisations... en faveur de

15 Alors que la Russie de B. Eltsine n'avait capté que 45 dollars par habitant de stocks d'IDE cumulés jusqu'en 1997, la Hongrie, pendant la même période, avait attiré plus de 1600 dollars d'IDE par habitant et en gros la moitié des IDE destinés aux pays en transition (hors la Chine). 
l'État et des privatisations de masse en faveur des insiders (employés et managers de l'entreprise) ${ }^{16}$.

a) Des variantes d'étatisation ont, en effet, paradoxalement été recensées en tant que privatisation. Il faudrait pourtant distinguer ne serait-ce que le transfert de propriété vers des collectivités locales, qu'il serait plus juste de désigner comme une propriété publique décentralisée, importante en Chine, notamment dans les zones rurales (Lemoine, 2003).

Nous ne discuterons pas ici de cette présentation pour le moins ambiguë de changements de forme de la propriété assimilés en bloc à des privatisations, probablement sous l'influence de l'anti-étatisme originel des préceptes dominants. Nous soulignerons plutôt la rationalité de ce classement du point de vue de notre sujet car ces privatisations directes en faveur de l'État accompagnent en général le changement de logique socio-économique des nouveaux dirigeants. Elles confirment que le parti-État n'était pas, jusqu'alors, le "principal" des rapports de propriété : il régnait au nom des travailleurs dans une relation mutuelle oscillant entre conflictualité ouverte et consensus et sans posséder les attributs d'un véritable propriétaire. Dans l'ancien système, comme nous l'avons souligné, les procédures de mise en faillite des entreprises ou de licenciement étaient exclues (sans reclassement préalable) même si le propriétaire réel (le "principal", collectif) n'avait pas de réels moyens de contrôle et de décision. Par les privatisations directes, au contraire, il s'agissait de faire émerger (même sans apport d'argent) une vraie propriété de l'État autorisant à la fois un changement du statut des travailleurs et de la logique socio-économique de la gestion de l'entreprise, désormais sommée de se plier à des contraintes "dures" de marché.

C'est là que réside l'une des sources de l'ambiguïté des "réfolutions": la radicalité du changement introduit par l'État n'a sans doute pas été perçue initialement par les populations concernées. Elles escomptaient plutôt une continuité par rapport à l'État-parti de l'ancien régime, certes dictateur mais aussi protecteur. Cette illusion d'une persistance de la protection sociale fut renforcée, dans les premières années de la transition, par les victoires électorales des excommunistes sous l'étiquette socialiste ou social-démocrate.

16 Nous ne débattrons pas ici de la circulation ultérieure (revente) des titres de propriété, des multiples et opaques échanges (dettes contre actions entre État et banques) et de l'émergence, notamment en Russie, des grands réseaux oligarchiques. Il faudrait également comparer les privatisations de masse et les formes de propriété des employés en Pologne (JArosz, 2001), soumises plus qu'ailleurs à des contraintes financières et marchandes dures. 
b) Les privatisations de masse en faveur des insiders (employés et managers des entreprises) ont été mises en œuvre dans la plupart des pays (de la Russie à la République tchèque en passant par les pays balkaniques) au début de la transition et selon des modalités différentes. Elles revenaient à attribuer à la population et aux travailleurs un pouvoir d'achat en parts d'entreprise sous forme de distribution de coupons (vouchers) permettant d'acheter des actions, de distribution gratuite ou quasi gratuite d'actions, d'octroi de droits prioritaires et de tarifs préférentiels pour l'acquisition de parts d'entreprise. Les modalités de la privatisation varièrent, du recours à divers types de fonds d'investissement jusqu' aux enchères directes. Mais la règle fut de donner le choix aux travailleurs entre plusieurs variantes de la privatisation dont celles qui assuraient une priorité aux insiders (le reste des parts allant à l'État ou étant destinées à des ventes ultérieures en faveur d'outsiders privés), formule largement dominante dans la Russie d'Eltsine.

Telle fut la deuxième source d'ambiguïté de ces "réfolutions" après les privatisations étatistes : les privatisations de masse exprimaient explicitement la reconnaissance du fait que l'ancienne propriété collective revenait de droit (donc prioritairement et gratuitement) à une échelle massive aux travailleurs et à la population. En Russie, notamment, le programme fort médiatisé de privatisation "en cinq cents jours" élaboré par l'académicien S. Chataline au début de la décennie 1990 insistait sur la "restitution" au peuple d'une propriété usurpée et y voyait entre autres un moyen de mettre fin à la criminalité souterraine (Favarel-Garrigues, 2003).

\subsubsection{L'enjeu du salariat}

\section{a) L'émergence difficile d'un salariat de type capitaliste}

La privatisation directe sans capital - par l'État et de masse - a contribué à supprimer toute forme de droit de gestion et d'autogestion des travailleurs associée à la propriété sociale. L'objectif était de faire émerger un statut de salarié impliquant la soumission à un vrai propriétaire des moyens de production, apte à licencier sur la base de critères privés de rentabilité marchande. La flexibilité des conditions de travail fut introduite dans les codes du travail à la fin de la décennie 1990, de la Russie à la Pologne en passant par la Serbie. Mais, lors de la première phase des "réfolutions", les privatisations directes avaient masqué cet objectif, faisant apparaître le changement des droits de propriété comme étant bénéfique aux travailleurs alors qu'il remettait en cause les droits de gestion de la propriété sociale au profit d'une logique privée, vite subordonnée à des rapports monétaires. 
En Pologne, par exemple, au travers de ce qu'on appela la commercialisation, l'État de la transition devint le véritable propriétaire privé en place et lieu des travailleurs, la logique de rentabilité entraînant la suppression des conseils ouvriers dans les entreprises concernées. La mise en liquidation des entreprises sur la base de critères marchands était dorénavant possible, de même que leur vente ultérieure à un vrai propriétaire privé, alors que les travailleurs avaient cru trouver dans la propriété étatique une certaine protection.

Ces deux dimensions se retrouvent dans les privatisations de masse :

Du point de vue des employés, le choix pragmatique de cette forme de privatisation visait au moins à défendre certains droits sociaux, spécialement le droit à l'emploi, contre des restructurations qu'auraient imposées des outsiders privés (l'État étant perçu comme moins dangereux).

Du point de vue des réformateurs, en revanche, il s'agissait d'abord de légitimer les privatisations aux yeux de la population ; ensuite et simultanément, de prouver aux institutions internationales qu'il y avait privatisation et donc rupture avec l'ancien système, ce qui conditionnait l'octroi de crédits ou le rapprochement avec l'Union européenne (UE). Par là même, un processus de polarisations sociales nouvelles et de concentration de la propriété s'engageait, notamment au profit des anciens directeurs d'entreprise ou de branche, sur fond de dispersion d'un actionnariat populaire dépourvu d'argent et de pouvoir, sauf celui de ralentir les restructurations (Clément, 2000). L'État devenu principal actionnaire (comme dans la forme étatiste extrême évoquée plus haut) pouvait désormais utiliser à sa convenance ses nouveaux droits de propriété pour avantager ses "clients" électoraux, rembourser ses dettes ou attirer de vrais investisseurs privés, nationaux ou étrangers.

\section{b) Le passé présent}

Lors des privatisations de masse, le non-paiement des salaires tout comme la non-fermeture d'entreprises en faillite se conjuguèrent avec des relations de troc, dominantes en Russie jusqu'à la crise de 1998 (Sapir, 1998 ; Zlotowski, 1998). Illustrant la survivance d'éléments de l'ancien système et des mécanismes de gestion couplés aux anciens "droits de propriété", ils représentent l'une des difficultés majeures de la privatisation et de la restructuration des grandes entreprises.

Dans la période post-stalinienne, en effet, quand les travailleurs furent libres 
de choisir leur emploi et donc de se mettre en quête de meilleures conditions de travail, des rudiments de marché du travail apparurent. Mais pour réaliser le plan (Lewin, 2002), attirer et garder les travailleurs dans les grandes entreprises, le revenu monétaire n'était pas un stimulant suffisant quand l'accès à nombre de services et produits de base, gratuits ou fortement subventionnés, nécessitait peu d'argent. La production et la distribution en nature par les grandes entreprises de biens et services, notamment gérés par les syndicats (logements, crèches, hôpitaux, produits fournis dans les magasins des entreprises...), jouaient un rôle essentiel de socialisation.

La perte de l'emploi implique celle de tous ces avantages difficiles à externaliser quand les budgets sociaux des municipalités et de l'État se réduisent sous la pression libérale et quand les salaires deviennent trop faibles pour acheter les biens et services aujourd'hui privatisés. Tout en se dégradant, les avantages sociaux liés à l'emploi ont partiellement subsisté dans les anciennes entreprises et, avec les lopins de terre, ont permis d'éviter une explosion sociale. En effet, durant l'étape initiale de la transition, l'emploi fut préservé (et donc le logement et autres avantages en nature) même en situation de faillite et de non-paiement des salaires. La montée du chômage apparent a ainsi été ralentie dans une phase où la comptabilité des entreprises et les prix se transformaient radicalement et où les entreprises, qui assuraient jusque-là une grande partie de la protection sociale sans subir de contrainte budgétaire, étaient de plus en plus soumises à des critères de rentabilité ${ }^{17}$.

C'est pourquoi notre désaccord, déjà évoqué, avec R. Motamed-Nejad (1997b) à propos du critère du surplus monétaire implique, corrélativement, une divergence importante avec son approche du salariat soviétique. Tout en distinguant, à juste titre, ce salariat de celui du capitalisme, il estime qu'il s'agit d'une "structure dominante" des systèmes "socialistes" (Yougoslavie exceptée). Une telle formulation n'a de sens, selon nous, qu'en cas de domination des relations monétaires et marchandes dans la gestion de l'emploi. Il ne s'agit ni de nier l'existence de revenus monétaires reflétant en partie des relations marchandes ni de minimiser les relations employeurs-salariés dans l'économie socialiste (Naville, 1970). Mais la thèse d'un salariat dominant suppose un marché du travail régulé par des mécanismes de prix affectant notamment les salaires. Comment expliquer alors les pénuries récurrentes de

17 C. Bettelheim (1970) a analysé de manière approfondie la mauvaise, voire la non-prise en compte des coûts dans le système soviétique. 
main-d'œuvre dans les grandes entreprises où l'intensité du travail était notoirement faible, au point que les "soviétologues" invoquaient le chômage caché ou le suremploi ? Ce constat n'est paradoxal que si l'on suppose l'existence d'un marché du travail ou de relations principalement salariales dans l'emploi, c'est-à-dire des relations où l'employeur peut imposer une intensification du rythme de travail sous la pression d'un licenciement et/ou d'une modulation du salaire. La prise en compte des relations de propriété impures impliquant la quasi-impossibilité de licencier fait ressortir, au contraire, la faiblesse des contraintes de type salarial pesant sur le rythme et l'allocation du travail. Bien plus que les revenus monétaires, les avantages sociaux hors salaire accompagnant l'emploi dans les grandes entreprises soviétiques permettaient, indépendamment de leur coût pris en charge par le système et non par les entreprises, d'attirer et de stabiliser la main-d'œuvre nécessaire à la réalisation des objectifs planifiés. Comparer la rentabilité des entreprises de type soviétique avec celle des entreprises engagées dans des relations de marché et n'assumant aucune protection sociale est un non-sens méthodologique. Les vestiges de ces anciens mécanismes et avantages sociaux expliquent pourquoi la restructuration capitaliste des grandes entreprises est extrêmement coûteuse et risquée (politiquement et socialement) pour le capital privé. Les relations de troc et les avantages sociaux non monétaires ont, pour la même raison, durablement persisté en Russie (et dans les pays issus de l'ex-URSS) au cours des années 1990. Hérités du passé, ils ont résisté à la régulation marchande, notamment dans le domaine de l'emploi.

Autrement dit, la lenteur et les formes contournées de la privatisation des grandes entreprises traduisent les contradictions essentielles de "réfolutions" confrontées au noyau dur du système passé. Certains auteurs (Walsh \& Wheland, 2001) jugent même "efficaces" les privatisations de masse comme étape initiale de la transition en ce qu'elles ont permis d'éviter des explosions sociales tout en accélérant des changements (suppression de la propriété sociale et de son filet de protection), voulus mais impopulaires.

Quels enseignements pouvons-nous tirer de tout ce que la transition remet en cause pour déterminer les critères pertinents permettant de cerner en quoi l'ancien système se différenciait du capitalisme émergent ?

\section{Du NOYAU DUR DE LA TRANSITION À L'ANCIEN SYSTÈME ET RÉCIPROQUEMENT}

\subsection{Ce Que La transition Remet En CAUSE}


Des "invariants" rapprochaient l'ensemble des pays se proclamant socialistes, de l'URSS à la Yougoslavie titiste, de Cuba à la Chine (au moins jusqu'à la fin des années 1980) ; ils sont remis en question par la transition vers le capitalisme, signe qu'ils sont, par essence, en contradiction avec celui-ci :

- la domination structurelle, socio-économique, juridique et idéologique, de la propriété sociale (dans ses diverses déclinaisons) par rapport aux différentes formes de propriété privée. Cette domination s'accompagne d'une critique explicite du capitalisme portée par les institutions (État, partis, syndicats) ; elle se traduit par des politiques fiscales et de crédit systématiquement favorables à la propriété sociale au détriment du secteur privé. Les modes de gestion de cette propriété ont pris des formes variées allant de l'étatisme le plus extrême à une décentralisation plus ou moins poussée ;

- le caractère structurellement non dominant des relations monétairesmarchandes est organiquement corrélé avec le caractère social de la propriété au plan macroéconomique. Il peut aussi s'analyser sous l'angle des fonctions de la monnaie et des prix dont l'objectif, précisément, est de subordonner le marché à des critères socio-politiques. D'où, généralement, l'absence de sanction marchande (licenciements et faillites) des déséquilibres, le rejet ou la marginalisation des marchés du capital et du travail. De même, la gestion de l'emploi et la formation des revenus monétaires incorporent des critères sociopolitiques dominants, valorisant symboliquement les travailleurs. Autrement dit, l'existence de catégories économiques telles que la monnaie, les prix, les revenus monétaires, le crédit ne signifie pas la prédominance des rapports marchands.

\subsection{LA DIVERSITÉ DES RÉFORMES DANS LA MÊME ESPÈCE DE SYSTÈME, NON CAPITALISTE}

Ces invariants sont dominants mais non exclusifs. Ils ont pu se combiner avec une vaste palette d'expériences et de réformes qui ont éloigné la plupart des pays du moule soviétique hypercentralisé. Prendre celui-ci comme seule norme revient à ériger en critère absolu ce qui fut contingent.

L'étiquette socialiste, discutée et discutable, n'est pas nécessaire de notre point de vue. Le fait de la refuser s'appuie sur le constat de relations d'oppression et d'exploitation (au sens d'une absence de contrôle social démocratique des conditions de production et de distribution) allant à l'encontre des idéaux socialistes. En tout état de cause, la cristallisation d'un appareil bureaucratique, renforcée par un système de parti unique, doit être analysée comme la réalité historique des pays du "socialisme réel" dont l'écart avec les 
idéaux proclamés a été et demeure contesté de l'intérieur comme de l'extérieur au nom même du socialisme.

Cette référence officielle au socialisme n'était pas neutre: les objectifs théoriques et émancipateurs du socialisme (ou de la critique du capitalisme) s'étaient transformés en une idéologie d'État sombrant dans l'apologie et la censure. Pourtant, ils ont également servi à ceux qui contestaient les rapports de domination existants et prônaient des réformes. Ce fut notamment le cas en Yougoslavie où les dirigeants titistes se réclamaient de Marx et de la Commune de Paris contre Staline et l'étatisme (Djilas, 1962 ; Kardelj, 1976), de même que les opposants de gauche au régime titiste, regroupés autour de la revue Praxis (Supek, 1973), arguaient des idéaux émancipateurs marxistes contre la bureaucratie du parti unique.

Le cas chinois peut être analysé comme une trajectoire spécifique d'entrée en transition (vers le capitalisme) sous l'étiquette communiste. Mais encore faudrait-il distinguer réforme du système non capitaliste chinois et " réfolution " instaurant la domination des critères capitalistes marchands. Un tel infléchissement est forcément lourd de contradictions sociales majeures et donc, aussi, de tensions au sein du parti unique. Pour l'instant, celles-ci sont contenues par une croissance vigoureuse, à son tour stimulée par le maintien de régulations étatiques et protectionnistes fort éloignées des préceptes libéraux qui l'emportent ailleurs. Il en résulte une sorte de dualisme social et idéologique dont les compromis, fluctuants, sont traités avec pragmatisme. Là comme ailleurs, la partie des élites au pouvoir la plus favorable à une rupture avec l'ancien système entend bannir les références aux valeurs du socialisme et réduire au minimum la protection sociale, ce que préfigurent sans doute les récentes modifications des statuts et de l'idéologie du Parti communiste chinois. La nature encore partielle de cette tendance exprime sans doute davantage la crainte du risque d'explosions sociales qu'un choix idéologique.

Notre postulat de la non-domination des rapports marchands n'implique pas une approche normative de la planification ou du centralisme de la gestion. Certes, la planification est contraire à la domination marchande. Pourtant, la forme de cette planification n'est pas prédéterminée ni en rien réductible à celle de l'URSS. Les réformes hongroises et yougoslaves de l'époque titiste, dont la dynamique et la temporalité sont toutefois différentes, ont introduit divers modes de coordination assurant d'importantes marges de responsabilité et de gestion décentralisée ainsi qu'un développement de la régulation marchande, amorcé en Yougoslavie en 1965-66 puis remis en cause au début de la décennie suivante. Mais la désétatisation des fonds d'investissement (distincts du budget 
de l'État) ou leur décentralisation vers des régions ou des républiques n'abolit pas en soi le caractère social de la propriété et la logique non marchande des critères d'allocation des investissements.

La non-domination des rapports marchands (du capitalisme) se traduit par l'absence d'un véritable marché du capital, les limites étroites de l'accumulation du capital et une ample protection des travailleurs contre la logique marchande salariale. Le socialisme de marché de la Yougoslavie titiste s'insérait dans ce cadre alors que celui de la Chine tend à s'y soustraire.

2.3. L'INTÉGRATION DES EXPÉRIENCES YOUGOSLAVES DANS LE SYSTÈME - UNE PROPRIÉTÉ DE TOUS ET DE PERSONNE

Même durant la phase du socialisme de marché (Samary, 1988) où s'est développée une sorte de propriété de groupe, les droits concernant l'embauche, y compris du personnel dirigeant, la structure des revenus, les investissements, etc. reconnus aux travailleurs yougoslaves s'étaient accrus, contrairement à ce qui se produit en Chine. Les droits dérivant de l'autogestion s'appliquaient également dans les co-entreprises (joint-ventures) ouvertes aux investisseurs étrangers, ce que ces derniers contestaient ou s'efforçaient de contourner. La tendance à des polarisations verticales au sein des entreprises et à des formes de privatisation capitaliste ${ }^{18}$ observée en Yougoslavie à la fin des années 1960 fut dénoncée comme frauduleuse et contraire à la Constitution. Les autorités réagirent par un coup d'arrêt au début de la décennie suivante en introduisant un nouvel ensemble de réformes avec, en particulier, la resocialisation du système bancaire. Le fait de déclarer illicite l'accumulation capitaliste est un indicateur fort de tensions, restées internes, d'un système non (encore) capitaliste. Notons, toutefois, que la critique du secteur bancaire n'avait pas uniquement des fondements économiques mais aussi politiques ${ }^{19}$. De même, contrairement aux thèses distinguant qualitativement la Yougoslavie titiste des années 1970 et les autres pays socialiste, les forces centrifuges qui y étaient à l'œuvre ont, jusqu'aux années 1980, été partiellement endiguées.

18On peut regrouper sous ce terme deux types de processus que les débats de cette époque dénonçaient comme contradictoire avec les principes socialistes du système : en premier lieu toute forme de réémergence d'un salariat que l'on peut aussi décrire comme forme d'autonomisation des structures de direction d'entreprise tendant à une gestion et appropriation privée du surplus, s'émancipant de fait du contrôle des autogestionnaires ; d'autre part, mais associée à la première tendance, les formes d'émergence d'une sorte de capital financier, accumulation de fonds gérés de fait par une osmose des directions d'entreprises et de banques orientées vers une logique de profit marchand dans cette phase des réformes (cf. Samary, 1988a).

19 Cette critique, de type nationaliste, provenait avant tout des dirigeants croates. Elle s'adressait à Belgrade - où se situaient la plupart des sièges sociaux des banques - et donc, implicitement, à la Serbie. En 1971, elle débouchera sur le mouvement politique dit "Maspok". 
Le maintien du caractère dominant de la propriété sociale a eu une influence décisive sur l'issue des tensions surgies à chaque étape. Celle-ci a évolué dans son contenu ou ses modalités de gestion. Telle que définie par la Constitution de 1974 en vigueur lors de l'éclatement de la Fédération yougoslave, elle représentait une propriété sociétale de tous et de personne. Dans leur phase ultime, les réformes titistes rejetèrent explicitement les précédentes variantes de la propriété sociale considérées comme restrictives, voire accusées d'usurper les droits collectifs. Ainsi, le propriétaire (en droit d'aliéner la propriété) ne pouvait être l'État, au plan fédéral ou républicain, en référence à l'étatisme de la première phase du régime (ou de l'URSS). Mais il ne pouvait pas non plus être incarné par les collectifs de travailleurs des entreprises car cela aurait équivalu à une propriété de groupe privant la société de son droit de regard, en référence, cette fois, aux conflits survenus entre 1965 et 1971 après l'introduction du socialisme de marché et l'abandon de la planification. Les moyens de production (l'entreprise) appartenaient à la société tout entière et ne pouvaient être aliénés sur la seule base des résultats marchands.

La propriété sociale était organiquement liée au statut des travailleurs, c'està-dire aux droits autogestionnaires (gestion des entreprises par les travailleurs eux-mêmes en vertu de leur droit de propriété). Ce statut excluait explicitement l'idée d'un rapport salarial, au point que le mot "salaire" avait, depuis les années 1960, disparu du vocabulaire et des comptes des entreprises. Bien ou mal réalisé, le partage sociétal de la propriété impliquait, partiellement, celui de la gestion, respectant le système de la protection sociale et des normes et obligations (qui ont évolué). L'allocation du surplus prenait en compte les besoins de l'appareil d'État (fiscalité alimentant le budget) distingués des besoins de la consommation collective. Cette dernière était financée par des contributions à des fonds spécifiés (logements, crèches, hôpitaux... à divers niveaux territoriaux) dont la gestion était partagée entre usagers, travailleurs, institutions publiques. Moyennant la couverture des coûts de production (dont les revenus individuels des travailleurs ne faisaient plus partie puisque considérés comme une composante du revenu global de l'entreprise autogérée), le paiement des impôts et autres contributions ainsi que les versements au Fonds d'amortissement), d'importantes marges de manœuvre-choix décentralisés - subsistaient dans l'allocation du revenu net à divers fonds de l'entreprise (revenus personnels, consommation collective, investissement...). Les normes et critères limitant les choix décentralisés en matière de gestion relevaient eux aussi de la propriété sociétale. C'est en son nom et pour assurer la cohésion et le développement de l'ensemble du pays qu'une part plus ou moins grande des investissements était centralisée et planifiée sous des formes 
évolutives ${ }^{20}$. Mais c'est même, paradoxalement, également en son nom qu'une décentralisation extrême fut tentée en 1965, l'hypothèse étant que l'augmentation des droits des autogestionnaires à gérer directement le surplus de façon décentralisée, sur la base des indications de marché permettrait de mieux satisfaire les besoins que les planificateurs. Et c'est encore au nom de la cohésion sociale du système et de la propriété que cette réforme fut remise en question dès $1971^{21}$ compte tenu des inégalités croissantes, perçues comme injustes par rapport au critère "à chacun selon son travail ".

Comme en URSS, en Pologne ou en Hongrie, les réformes furent entreprises en Yougoslavie par le parti-État, interrompues du fait des tensions sociales, puis modifiées après le musellement par la force des contestataires. Tel fut notamment le scénario qui mit fin au " socialisme de marché " introduit en 1965. La répression du mouvement d'étudiants et d'enseignants de juin 1968, la remise au pas parallèle des syndicats et des mouvements nationalistes à la fin de la décennie, furent le prélude à un nouveau tournant institutionnel. Celui-ci prit partiellement en compte les revendications_parfois contradictoires des uns et des autres mais sans véritable débat puisque les courants politiques indépendants, se réclamant ou non du socialisme, n'eurent pas le droit à la parole. Cette combinaison de répression et concessions, propre au titisme, ne pouvait créer de véritable cohérence. Il s'en suivit un nouveau système de planification autogestionnaire et une confédéralisation visant à contenir les tensions, mais où les acteurs (autogestionnaires et pouvoirs républicains) utiliseront les marges croissantes d'autonomie accordées bien davantage que l'incitation à s'associer. La nouvelle planification autogestionnaire sur base contractuelle ne sera donc pas à même de réguler des dépenses d'investissement décentralisées et stimulées par la transformation de la gestion bancaire favorable à l'endettement. La confédéralisation du système accentuera, quant à elle, le rôle croissant du cadre républicain pour la politique fiscale et les échanges commerciaux. Mais le creusement des écarts régionaux et l'endettement extérieur sonnèrent le glas de ces réformes au tournant des années 1980. Ils étaient dus à des facteurs internes

20 Si la planification a changé de forme et d'instruments dès les années 1950 par rapport à l'hypercentralisme de type soviétique, elle n'a été remise en cause que dans la phase limitée de 1965 à 1971 dite de socialisme de marché : les fonds centraux d'investissements de la période précédente y avaient été démantelés, l'essentiel du surplus des entreprises étant alors laissé aux fonds d'investissement des entreprises ou affecté à un système bancaire obéissant dans cette phase là à une logique de marché. Après les conflit sociaux surgis entre 1968 et 1971, les fonds bancaires furent renationalisés et une nouvelle forme de planification contractuelle établie, codifiée par des amendements constitutionnels (Samary, 1988a).

21 Dès 1971, Tito lance une « révolution culturelle » qui vise à mobiliser les travailleurs contre les directions des entreprises et des banques, tout en réprimant toutes les dissidences politiques et syndicales. Entre 1971 et 1974, des amendements constitutionnels majeurs vont codifier de nouvelles définitions de la propriété sociale, appuyées sur une restructuration des entreprises et des banques (Samary, 1988a). 
(hausse de l'endettement et du gaspillage joints à l'absence de cohérence politique et socio-économique d'ensemble), brutalement aggravés par des facteurs internationaux (deuxième hausse pétrolière puis montée des taux d'intérêt prélevés sur la dette et tournant libéral des institutions financières internationales à la fin de la décennie 1980). [[On va raccourcir ici, puisqu'on a rallongé avant, et qu'on peut préciser le thème après]]

Les transformations de la propriété opérées en Yougoslavie à partir de 1989 vont être introduites comme une réponse à la crise économique visant à rétablir une cohérence de marché. Elles rejoignent ainsi pleinement les caractéristiques de la "réfolution" et de la nouvelle logique à l'œuvre (sous des formes diverses) dans les privatisations de masse (voir supra). Mais en Yougoslavie plus que dans les autres pays socialistes, les travailleurs jouissaient de droits explicites de propriétaires collectifs. Un "troc" leur sera donc proposé : l'échange gratuit (ou quasi gratuit) de leurs anciens droits et avantages sociaux non monétaires contre des "actions"... laissant, un temps, perdurer l'illusion qu'ils restaient propriétaires et donc maîtres de leur emploi. Ce sont là des caractéristiques générales des pays en transition. Pourquoi faudrait-il, dans ce cas, dissocier la Serbie? C'est ce que nous allons examiner à présent en intégrant dans l'analyse une comparaison avec la Slovénie et le traitement qu'elle a réservé à la propriété sociale yougoslave.

\section{DE LA TRANSITION YOUgOSLAVE À LA TRANSITION SERBE COMPARÉE À LA TRANSITION} SLOVÈNE

\subsection{De la transition yougoslave à l’éclatement de la Fédération}

Dans le contexte international et interne de la crise du socialisme réellement existant, éclata la crise de dette extérieure en devises de la République socialiste fédérative de Yougoslavie, une dette qui a plus que doublé entre 1977 et 1981 pour atteindre 21,1 milliards de dollars et demeurer proche des 20 milliards de dollars au cours de la décennie, en dépit des politiques d'austérité conditionnant le rééchelonnement de la dette (Samary, 1988a) ${ }^{22}$. Les résistances qui s'opposèrent aux divers plans de remboursement de la dette tant au plan des entreprises que des républiques,

22 L'absence de cohérence et de régulation de la politique d'endettement et de commerce extérieur, évoquée plus haut pour les années 1970 ont été les facteurs internes de cette dette. Mais elle fut aggravée par la hausse des taux d'intérêt sur la dette au cours de la décennie 1980 et par le mouvement des prix relatifs des biens échangés. 
nourrirent l'hyperinflation des années $1980{ }^{23}$. Celle-ci donnera du poids aux solutions libérales, dans ce pays comme ailleurs. Les réformes du dernier gouvernement yougoslave d'Ante Markovic en 1989 observent la doctrine libérale tant en ce qui concerne la politique dite de stabilisation (contre l'inflation) que les changements structurels. Seuls ces derniers nous occuperont ici.

\subsubsection{Les réformes d'Ante Markovic de 1989: le début de la transition yougoslave}

Les réformes furent élaborées et acceptées par l'ensemble des représentants des républiques et provinces qui siégeaient dans les instances fédérales sur des bases égalitaires et bénéficiaient du droit de veto. À cette époque, Slobodan Milosevic était déjà au pouvoir en Serbie. Formé à la gestion bancaire, il joua un rôle actif dans la préparation des réformes. Selon Susan Woodward (1995, pp. 106-107), qui évoque les travaux précédant l'adoption de la loi, " les propositions avancées par la "commission Milosevic" en mai 1988 ont été rédigées par des économistes libéraux et directement tirées du livre de recettes du FMI ". Elles avaient pour objectifs la suppression des droits autogestionnaires et l'émergence, à terme, d'un rapport de propriété capital/travail salarié. Mais il était impossible de les imposer directement ou après une consultation démocratique étant donné la popularité de l'autogestion parmi les travailleurs

La première loi fédérale sur les privatisations d'août 1990, qui fait l'objet d'adaptations dans certaines républiques en 1990-1991, prive la propriété sociale de sa prépondérance en mettant sur le même plan toutes les formes de propriété. Parallèlement, elle démantèle l'aspect sociétal de la propriété en donnant aux entreprises autogérées le statut de vrais propriétaires, en droit de privatiser "leur" entreprise. L'atomisation des entreprises, nécessaire à la compétition marchande, remet également en question la planification autogestionnaire. Mais la forme du processus engagé permet de maintenir les droits autogestionnaires là où ils étaient le plus concrets, à savoir dans l'horizon borné de l'entreprise. Chaque collectif d'entreprise devait commencer par faire évaluer "son" capital social, étape ouvrant la voie à une privatisation ultérieure.

23 Les années 1980 sont celles d'une politique restrictive d'ajustement extérieur, d'une stagnation du produit social, etc. En ont résulté des tensions politiques croissantes qui permettent de mieux comprendre la suite, c'est-à-dire la montée des nationalismes et, en fin de compte, l'éclatement de la Yougoslavie. 
La première phase des réformes consistait donc à revenir, sans le dire et sans la moindre consultation populaire, vers la propriété de groupe, pourtant explicitement exclue par la Constitution de 1974. La "réfolution" était en marche, contournant le tabou de l'autogestion.

\subsubsection{Quel(s) État(s) bénéficiaire(s) et garant(s) des privatisations? Vers} l'ethnicisation de la propriété

La crise socio-économique et politique d'ensemble affecta la crédibilité de l'État fédéral (Horvat, 1992). Le nationalisme post-yougoslave (Kubli, 1998) fournissait une double source de légitimation à une transition en deux volets : privatisation et éclatement de la Fédération. Aux anciens avantages de l'autogestion socialiste allaient se substituer ceux du communautarisme pour les catégories les plus fragilisées (travailleurs et paysans attendant de "leur" État qu'il protège leur emploi et leur terre) et la proclamation du droit de quitter la Fédération (autodétermination) consolidant l'ethnicisation de la propriété.

Ce droit à l'autodétermination était ambigu : s'agissait-il d'un droit des peuples constitutifs de la Yougoslavie ou d'un droit des républiques, sachant que ces dernières comportaient généralement de fortes minorités ou plusieurs communautés reconnues comme peuples? Sans pouvoir en discuter longuement ici, disons que le droit à l'autodétermination fut interprété de façon conflictuelle et évolutive, en quelque sorte "à la carte" : droit de l'État et procédures fréquentes de consultation des citoyens par référendum là où la communauté demandant l'autodétermination était majoritaire ; droit des peuples au sens ethnico-national prévalant partout où la communauté concernée était minoritaire et éclatée dans plusieurs États, chacune refusant aux autres ce qu'elle exigeait pour elle-même (Morokvasic, 1992 ; Samary, 1994, 1999c).

Dans la décennie 1990, l'éclatement de la Fédération eut pour enjeu l'établissement de frontières contrôlables par les nouveaux États-nations émergents. Ils entendaient s'approprier les richesses (et les devises tirées de l'exportation) correspondant à ces territoires et, parallèlement, assurer leur insertion en ordre dispersé dans la construction européenne et la mondialisation. Les conflits et guerres dans l'espace yougoslave, notamment en Bosnie (Bougarel, 1996) et au Kosovo (Roux, 1999 ; Batakovic, 1993), relèvent, au plan intérieur, de cette logique de contrôle des territoires, tributaire de majorités ethniques. Cependant, toutes les communautés nationales de l'ancienne Yougoslavie n'étaient pas dotées des mêmes statuts 
ni des mêmes relais institutionnels pour faire valoir leur cause.

Les Albanais et les Hongrois de Yougoslavie n'étaient pas considérés comme des nations (au sens ethnico-national) mais comme des nationalités (terme visant à éviter celui de minorité) dont l'État de référence se situait à l'extérieur : ils ne jouissaient pas du droit à l'autodétermination. En revanche, les provinces de Voïvodine et du Kosovo, qui faisaient explicitement partie de la République de Serbie (art. 1er de la Constitution de 1974), bénéficiaient de droits qui se sont progressivement imposés dans la pratique, notamment un droit de veto dans les instances fédérales, et de représentants dans ces mêmes instances. Slobodan Milosevic "résoudra" les incohérences de ce statut par la manière forte en rétablissant le pouvoir de Belgrade sur les provinces et, partant, sur les mines du Kosovo.

Autres entités fragiles, la Bosnie-Herzégovine et la Macédoine, dont le statut de république et les droits nationaux de leurs peuples respectifs avaient été consolidés par le titisme et le cadre yougoslave, bientôt jugés " artificiels" et donc contestés, en leur sein même ${ }^{24}$ et par leurs voisins. Les représentants de ces deux Républiques s'efforcèrent désespérément de maintenir un cadre yougoslave de compromis entre les projets de plus en plus confédéralistes de la Slovénie et de la Croatie et la recentralisation de la Fédération au profit de la majorité serbe, préconisée par Belgrade. La sécession de la Slovénie et de la Croatie en juin 1991 les plaça devant un dilemme : l'indépendance au risque de confrontations ou le tête-à-tête avec la Serbie dans une Yougoslavie tronquée.

Le Monténégro, quant à lui, fut tiraillé entre plusieurs logiques mais il resta attaché, à défaut d'indépendance, à la consolidation titiste de son autonomie et à ses prérogatives économiques - qu'il n'exerça vraiment qu'après 1997 (lois de privatisation, système monétaire, régime douanier propres) - comme base de négociation de son alliance avec la Serbie, résistant à toute variante d'État unitaire ${ }^{25}$.

Slobodan Milosevic s'efforça de contrôler le territoire le plus vaste possible en jouant sur tous les registres idéologiques. Il se présenta en défenseur des minorités et intérêts serbes au Kosovo, en Croatie et en Bosnie (Popov, 1998) tout en négociant avec le dirigeant croate F. Tudjman le

24 Les Serbes de Bosnie-Herzégovine refusaient que cette République deviennent indépendante et les Croates qui y vivaient revendiquaient le rattachement de l' "HerzegBosna " à la Croatie.

25 Depuis, la situation a changé : les partisans de l'indépendance ont pris plus de poids mais aucune majorité ne se dessine dans un sens ou dans l'autre. Pour une analyse de l'évolution du Monténégro, voir l'article d'Amaël Cattaruzza dans ce numéro. 
partage de la Bosnie et en faisant vibrer la corde "yougoslave" : celle-ci était essentielle pour conserver l'intégrité d'une Serbie multiethnique, consolider l'alliance avec le Monténégro et ne pas s'aliéner l'armée soucieuse de voir perdurer l'État yougoslave, source de ses privilèges.

Pourtant, l'abrogation des dispositions de la Constitution de 1974 sur la propriété sociale et le statut des provinces de Serbie marqua le début de son éclatement. L'arrêt de mort de la Yougoslavie titiste date de juin 1991 lorsque la Slovénie et la Croatie, gouvernées par des partis indépendantistes arrivés au pouvoir en 1990, proclamèrent leur indépendance, entérinée par l'UE à l'automne 1991 et, juridiquement, en janvier 1992. La transition relevait désormais des nouveaux États indépendants.

\subsection{LA TRANSITION SERBE ENTRE ENJEUX TERRITORIAUX ET SANCTIONS AU COURS DE LA} DÉCENNIE 1990

Après les sécessions, la Serbie (comprenant les provinces du Kosovo et de la Voïvodine) forma, avec le Monténégro, la République fédérale de Yougoslavie (RFY) le 27 avril 1992 en place et lieu de l'ancienne République socialiste fédérative de Yougoslavie (RFSY). La population ne fut pas consultée.

La perspective d'une reconfiguration violente des frontières des républiques sur des bases ethniques troubla l'évolution de la transition et les rapports internationaux : par son rôle dans les guerres ou, selon le cas, dans les plans de paix, Belgrade s'inséra dans la Realpolitik des grandes puissances, que l'embrasement des Balkans inquiétait. Tout cela ne fut pas sans effet sur les différenciations sociales et politiques internes (Lazic, 1999 ; Goati, 1995).

Mais dans l'ensemble, si l'on prend pour critère de comparaison la Slovénie de façon à intégrer la part de l'héritage de l'autogestion dans le processus de transformation, le principal facteur de différenciation du modèle serbe de privatisation s'avère être le contexte de guerres et de sanctions (1992-1996). La recherche par le régime d'un soutien populaire contre les sanctions pesa sur les privatisations à plusieurs égards : hyperinflation accompagnée de création monétaire, suspension explicite de toute restructuration pendant les sanctions, mais aussi tensions avec l'opposition autour de la privatisation des médias notamment.

Si l'on veut "gommer" l'effet de la guerre et des sanctions pour comparer 
les politiques de privatisation, il faut examiner ces dernières avant 1992 et après 1996. On peut y distinguer quatre phases dans les années 1990 (Privatizacij u Srbiji, 1997, 2001; The laws on enterprises and privatisations, FRY, 1998).

- Entre 1989 et 1991, la première vague de privatisation en Serbie se déploie dans le cadre de la loi fédérale de décembre 1989, modifiée en août 1990. Sur 7500 entreprises soumises à la loi de transformation de la propriété, 3500 relèvent de la propriété sociale, 3500 sont mixtes (combinant propriété d'État et sociale) et 500, appartenant à des secteurs stratégiques, sont étatiques. En douze mois, la transformation touche 1220 entreprises en propriété sociale, soit quelque $33 \%$ de leur nombre total.

- Entre 1991 et 1994, une deuxième phase de privatisation survient dans le cadre de la nouvelle loi serbe, adoptée en août 1991 après l'éclatement de la Fédération. À l'origine, elle se voulait particulièrement contraignante et rigoureuse en ce qui concernait l'évaluation, obligatoire, du capital et les conditions d'achat des parts réservées aux employés. Mais l'hyperinflation de 1992-1993 et la guerre provoquent des distorsions considérables. Au total, en 1994, 2000 entreprises sont engagées dans le processus de transformation dont certaines relèvent directement de la nouvelle loi et d'autres, des précédentes lois.

- Entre 1994 et 1997, la troisième phase des privatisations se caractérise par une réévaluation dans le but (officiel) d'annuler les gains acquis par l'achat de parts d'entreprises dans un contexte d'hyperinflation, c'est-à-dire de sous-évaluation du capital social. Cette réévaluation imposée se traduit par l'annulation de la privatisation de 436 entreprises. Le nombre d'entreprises privatisées ne change pas entre 1995 et 1997.

Une nouvelle loi de privatisation est préparée dans le contexte de levée des sanctions contre le régime de S. Milosevic étant donné sa participation aux accords de Dayton qui mettaient fin à la guerre en Bosnie (1995).

- Entre 1997 et 2000, la nouvelle loi sur la transformation de la propriété, qui vise à accélérer les privatisations, est adoptée en juin 1997. Elle prévoit de transférer gratuitement ou à un tarif très préférentiel jusqu'à $60 \%$ du total des parts de l'entreprise à ses actuels et anciens employés ainsi qu'aux citoyens travaillant dans les services publics, aux retraités ou aux paysans. Trois modèles sont proposés, incluant l'échange de dettes contre actions. En 
trois ans, la loi s'applique à 786 entreprises représentant $34 \%$ de l'ensemble des transformations de la propriété sociale.

Nous ne pouvons traiter ici de questions telles que les montages financiers, les rapports clientélistes et autres formes opaques de privatisation et de corruption ayant gangrené le régime de Milosevic. Notons, toutefois, qu'il n'en avait pas l'exclusivité même s'ils étaient très répandus. Son opposition n'a pas toujours été en reste et la plupart des autres pays en transition ont connu des scandales du même ordre.

Les privatisations légales ont été très favorables aux travailleurs. Mais la comparaison avec les privatisations de masse ayant eu lieu ailleurs, notamment dans la Slovénie voisine, montre qu'il s'agit une fois de plus d'une caractéristique générale de la première phase de la transition ne justifiant pas, à nos yeux, de faire de la Serbie un cas à part.

\subsection{La comparaison de la Serbie et de la Slovénie}

De tous les pays socialistes, la Slovénie, qui bénéficiait des meilleurs acquis relatifs du passé titiste, s'est le plus éloignée des formes dictatoriales et centralistes de l'ancien régime de parti unique (Félice, 1995; Vukadinovic, 1999, 2000, 2001) ${ }^{26}$. Mais il lui fallut plus de trois ans pour dégager un consensus autour des lois de privatisation: une grève générale bloqua les mesures radicales que le premier gouvernement de centre-droit voulut prendre pour accélérer la transformation de la propriété sociale sur les conseils de J. Sachs (1991). Ce projet fut contesté par le ministre de l'Économie, J. Menciger, qui démissionna de ses fonctions pour exprimer son désaccord. Comparativement aux autres pays en transition, les préceptes libéraux firent l'objet en Slovénie de résistances explicites, scientifiques (Menciger, 2000, 2002) et démocratiques ${ }^{27}$. Elles furent pourtant combattues par la Commission européenne en dépit des succès de la Slovénie dont le PIB par habitant est proche de celui de l'Espagne et supérieur à ceux de la majorité des autres PECO candidats. Au vu des résultats obtenus, la transition gradualiste de la Slovénie, opposée à la thérapie de choc choisie en Serbie, fait, aujourd'hui, plutôt figure de modèle.

26 Sur la rupture de la RSFY avec Moscou en 1948, les réformes yougoslaves et la place particulière de la Slovénie, voir également Derens \& SAMARY, 2000.

27 Nous n'adhérons pas, de ce point de vue, à la méthodologie d'A. Slim (1999) qui analyse la violence de l'accumulation primitive dans les pays balkaniques en y agrégeant, selon un critère géographique discutable, la Slovénie. Sous l'angle des trajectoires, celle-ci se rattache plutôt à l'Europe centrale. 
Après ces premiers mouvements de contestation, l'abolition de la propriété sociale prit en Slovénie deux formes. D'un côté, une étatisation de secteurs-clés, notamment des services publics et des banques, et de grandes entreprises assurant l'essentiel des emplois (métallurgie, télécommunications); d'autre part, une privatisation des entreprises en propriété sociale où le choix des procédures et de la répartition revenait majoritairement aux insiders. La transformation de la propriété soumettait en effet les collectifs des entreprises à des normes dans le cadre desquelles un choix demeurait possible pour $40 \%$ du capital social : soit les insiders (managers et employés) décidaient d'acheter cette part eux-mêmes à un tarif préférentiel, soit ils se tournaient vers des acheteurs extérieurs. Pour le reste, $10 \%$ étaient attribués à divers fonds sociaux (pour les retraites et le développement), $20 \%$ étaient distribués gratuitement aux travailleurs via un système de coupons leur permettant d'acheter des actions de n'importe quelle entreprise (vestige de l'ancien caractère sociétal de la propriété sociale), $10 \%$ étaient affectés à un Fonds de restitution (aux anciens propriétaires) et les $20 \%$ restants alloués au Fonds de privatisation.

Autrement dit, $70 \%$ (contre $60 \%$ en Serbie sous Milosevic) du capital social ont été vendus ou distribués en fonction de priorités sociales, par une reconnaissance $\mathrm{du}$ fait qu'il s'agissait d'un patrimoine sociétal issu fondamentalement du travail. On peut inclure dans cette catégorie, outre la part correspondant aux fonds sociaux, celle assortie de conditions préférentielles que les collectifs d'entreprise pouvaient matérialiser s'ils le souhaitaient (ce fut généralement le cas) et celle destinée à l'ensemble des travailleurs sous forme de droits gratuits sur n'importe quelle entreprise ; 20\% (30\% en Serbie) seulement furent réservés au Fonds de privatisation et $10 \%$ à la restitution.

L'État slovène s'est, de surcrôit, donné les moyens d'une politique industrielle et financière visant à soutenir et non pas à asphyxier, comme dans beaucoup d'autres pays, les grandes entreprises. À la fin de 1998, l'État contrôlait, outre les secteurs stratégiques évoqués, $40 \%$ du capital des entreprises privatisées tout en subventionnant les entreprises publiques par l'entremise d'un Fonds de développement. Jusqu'au tournant du millénaire, il a cherché à conserver le contrôle du système bancaire dans le but de maîtriser sa politique de crédit (CDC IXIS, Flash Marché émergents, 05.02.2002). Mais cela lui a valu les reproches répétés de la Commission européenne à laquelle il céda progressivement en vue de l'adhésion du pays à l'UE : il autorisa donc un accroissement de la part du capital étranger dans les banques de $16 \%$ en 2001 à $41 \%$ fin 2002.

Sur la base de cette comparaison, la logique veut que si l'on considère la 
Slovénie de la fin des années 1990, dotée de ces lois et caractéristiques, comme un pays en transition, alors la Serbie l'est aussi.

3.4. Octobre 2000 en Serbie et la nouvelle phase de la transition : vers de vrais SALARIÉS

\subsubsection{Début ou tournant de la transition?}

Le ministre serbe des Finances, Aleksandar Vlahovic, évoquait en février 2001 (www.serbia.sr.gov.yu consulté en mai 2003) "le début de la transition " à la "suite de la victoire de la démocratie ". Et il ajoutait que "la loi de privatisation [de l'ancien régime] était une régulation qui n'assurait pas la transition vers une économie de marché ". C'était au moins souligner l'objet des lois récentes : assurer ce que nous appellerons l'accomplissement (et non le début) de la transition. La question était de savoir comment.

Du fait des convictions libérales de l'opposition au régime de Milosevic et de la pénurie de capitaux nationaux, les capitaux étrangers furent considérés comme la clé des moyens financiers recherchés. Le G17 Plus, groupe rassemblant les économistes de l'opposition (ultérieurement constitué en parti), avait dressé en juin 1999 un bilan chiffré des dommages économiques " collatéraux" produits par 77 jours de bombardements (G17 Plus, 1999). Il estimait les dégâts directs et indirects (sur dix ans) à près de 30 milliards de dollars. Ce "bilan final", qui est controversé, exprimait l'espoir que les compensations versées pour les dommages de guerre permettraient à la Serbie de se moderniser. L'aide occidentale rétribuerait également, pensaiton, l'éviction de S. Milosevic et la mise en ouvre de réformes radicales. Bien que ces réformes aient débuté en 1991 et que S. Milosevic ait été transféré à La Haye le 28 juin 2001, cet espoir fut déçu ${ }^{28}$.

\subsubsection{Les nouvelles lois de privatisation : priorité aux outsiders}

Les réformateurs serbes ont eux-mêmes présenté (Jugoslovenski Pregled/Yugoslav Survey, 2001) les grands traits des mesures alors introduites :

28 Notons toutefois que, même s'ils furent moins importants qu'espéré, le pays bénéficia de dons et, surtout, de l'effacement des deux tiers de sa dette auprès du Club de Paris et, tout récemment, de $62 \%$ de sa dette auprès du Club de Londres, soit de conditions que l'on peut qualifier d'exceptionnelles. Peut-être les attentes étaient-elles démesurées compte tenu de l'état économique dramatique de la Serbie suite à dix années de gabegie et de guerres, ce qui ne signifie pas que la communauté internationale doive interrompre son aide, quelle que soit la forme qu'elle puisse prendre. Nous pensons même qu'elle devrait nettement l'accroître. 
1) une privatisation obligatoire et soumise à des contraintes de temps (délai de quatre ans) ;

2) l'abaissement, par diverses procédures, du poids des insiders qui prédominaient antérieurement : la règle générale est que $70 \%$ au moins du capital soit vendu et qu'un maximum de $30 \%$ soit distribué parmi les travailleurs et/ou citoyens (cette proportion pouvait être encore réduite si la partie destinée à être vendue ne l'avait pas été dans le délai imparti de quatre ans) ;

3) la recherche d'un capital nouveau (étranger et national) pour restructurer les entreprises existantes ;

4) la recherche d'une structure claire de propriété (privée et étatique);

5) assurer la propriété contre le risque ;

6) s'en remettre au gouvernement pour qu'il traite les questions sociales de façon à garantir aux investisseurs des conditions stables et égales sur l'ensemble du territoire (refus de procédures trop décentralisées).

\subsubsection{L'accentuation générale des critères libéraux}

Les nouvelles règles adoptées se fondent sur des critères normatifs (ajustés) de l'école des droits de propriété qui intégrent le bilan des privatisations de masse (Blanchard, 1997 ; Nellis, 1999 ; Sachs \& Woo, 1994). La lenteur des privatisations et des restructurations est imputée au poids des insiders et le but, désormais, est une restructuration protégeant l'investisseur du risque. Fait significatif de l'ajustement de l'approche standard, la propriété de l'État, qui n'est pas exclue, devrait être aussi "claire" que celle d'un propriétaire privé. C'est dire que l'État est désormais considéré comme un outsider capable d'imposer une discipline de marché et de mener une politique attractive pour l'investisseur étranger, au plan fiscal et social.

Les nouvelles lois serbes reflètent la radicalisation de la transition dans le sens du libéralisme depuis la fin de la décennie 1990. En témoigne l'adoption, de la Pologne à la Russie, de nouveaux codes du travail similaires à ceux de la Serbie. L'accent désormais mis sur les outsiders suppose qu'ils seront moins sensibles aux pressions des employés. Cette caractéristique ne marque donc pas le début, mais une nouvelle phase des politiques de privatisation d'inspiration néo-libérale. Les privatisations de masse avec poids prépondérant des insiders loin d'avoir été l'apanage de la Serbie de Milosevic ont été utilisées par des pouvoirs qui se sont réclamés explicitement du néo-libéralisme, comme la république tchèque de Vaclav Klaus. Et celui-ci a été en réalité confronté, comme Slobodan Milosevic et les autres dirigeants de ces réfolutions, aux mêmes difficultés de légitimation sociale des privatisations des grandes 
entreprises et au même manque de capital. Ceci explique pourquoi, derrière des discours se réclamant de Margaret Thatcher, les restructurations des grandes entreprises tchèques s'avérèrent fort limitées jusqu'en 1998 et recouvrir des montages financiers illustrant les liens opaques entre nouveaux pouvoirs, banques et managers pendant la première phase de cette transition. On peut considérer que s'ouvre une deuxième phase de la transition, quelle qu'en soit la date, lorsqu'elle devient plus explicitement capitaliste : il s'agit alors d'exiger un apport réel de capital, à son tour en mesure d'imposer aux employés le rapport de subordination et de gestion propre au salariat capitaliste. Il apparaît clairement dans cette deuxième phase qu'un tel rapport social et apport de capital s'appuient fondamentalement sur des outsiders supprimant radicalement les vestiges du système antérieur.

Ces transformations micro-économiques, s'articulent sur celles qui affectent, au plan macro-économique, les financements et le rôle de la monnaie, sur la base des réformes imposées par les pouvoirs publics. L'économie est alors soumise aux critères et mécanismes privilégiant les marchés financiers, tandis que s'imposent les contraintes d'austérité budgétaire propres au consensus, pourtant contesté, de Washington (Stiglitz, 1999, 2001 ; Sapir, 1999). Ainsi, l'Union européenne, impose aux nouveaux membres, une intégration aux mécanismes de change et aux critères d'adhésion préparant à l'euro. Ainsi, même s'il n'est pas possible d'envisager de passage rapide à l'euro pour tous, s'impose une approche monétariste pourtant contestée ${ }^{29}$. Les déficits budgétaires doivent se financer par l'émission de titres poussant à une hausse des taux d'intérêt dommageable à l'investissement et à la croissance. Parallèlement, la priorité accordée aux financements privés s'accompagne d'une politique fiscale d'attractivité des capitaux qui réduit encore les capacités d'intervention autonome des pouvoirs publics. Dès lors, pour ne pas creuser les déficits publics, ce sont les dépenses de cohésion sociale et d'éducation qui sont sacrifiées, les privatisations étant censées être plus efficaces, notamment dans tous les services, qu'ils soient bancaires ou d'éducation.

En effet, dernier volet de ces transformations d'ensemble, les Accords généraux sur le commerce des services (AGCS) négociés dans le cadre de l'OMC autorisent une pénétration massive des capitaux étrangers dans les systèmes

29 L'interprétation monétariste de l'inflation est loin de faire consensus même parmi les économistes libéraux. Selon elle, seule la création monétaire cause l'inflation. Cette thèse est si peu admise au sein du Système européen des banques centrales, que la lutte contre l'inflation s'y appuie officiellement sur plusieurs « piliers » (et non pas seulement sur le contrôle de la masse monétaire). La FED des Etats-Unis a elle-même remis en cause le monétarisme après 1981, et sait abondamment jouer sur divers objectifs et instruments de politique monétaire. Nombreux sont les économistes (dont le prix Nobel Joseph Stiglitz, 1999, 2002) qui soulignent le caractère contre-productif d'une hausse des taux d'intérêt visant à contrer l'inflation et à attirer des financement par titres de la dette publique quand une économie a besoin de se restructurer en créant massivement des emplois. 
bancaires : leur part était en 2002 majoritaire dans tous les PECO (hors Slovénie) dans des proportions allant de $60 \%$ environ (en Lettonie ou en Roumanie) à plus de $90 \%$ (en Estonie). Cette logique s'inscrit dans le durcissement des conditions de crédit dont le critère risque d'être le rendement à court terme. Ce critère est-il pertinent pour les besoins fondamentaux du développement, notamment l'éducation? Est-il en mesure d'aider à une restructuration de l'économie selon des critères d'efficacité visant l'élévation du bien-être et de l'éducation de la grande masse des populations ? Est-il porteur de développement ou simplement d'une croissance (mesurée par les PIB) sans justice sociale (Sen, 1999, 2000).

\subsubsection{Les " réfolutions" ont-elles été efficaces?}

La remise en cause de la gestion de l'économie par l'ancien parti unique signifie-t-elle pour autant que l'émancipation du financement des pressions politiques et sociales est un gage d'efficacité et de démocratie ? Alors que le 5 octobre 2000 s'était signalé par une puissante mobilisation populaire dans les bastions mêmes de l'ancien régime, le bilan d'une politique qui a laissé exploser le chômage au-dessus de $30 \%$ se reflète déjà dans le fort taux d'abstention ayant provoqué l'invalidation de plusieurs scrutins en 2003 et 2004 et dans l'amplification du vote en faveur de Vojislav Seselj, le candidat de l'extrême droite xénophobe et populiste. La Serbie s'aligne ainsi sur les récents résultats électoraux des Balkans occidentaux, de la Bosnie à la Croatie, mais également sur ceux de la Pologne et de la Hongrie où la montée des votes xénophobes suscite une même inquiétude. En somme, les pages noires ou les mythes du passé, exploités par les nationalismes, continuent d'alimenter des peurs ancrées dans les incertitudes socio-économiques de l'avenir.

Malgré une trajectoire spécifique, l'écart entre les attentes populaires et les réformes engagées est au moins aussi grand en Serbie qu'ailleurs. Pourtant, le test d'efficacité et de démocratie de ces réformes ne serait-il pas qu'une part significative des populations concernées ait le sentiment qu'elles sont bénéfiques et qu'elles sont le fruit de leur libre choix ?

\section{EN CONCLUSION : DE LA CRISE YOUgOSLAVE À LA CONSTRUCTION EUROPÉENNE}

Les causes profondes de la désintégration yougoslave persistent, à savoir l'objectif d'une appropriation des territoires et des richesses sur des bases ethniques. La fiction d'un État unitaire ${ }^{30} \mathrm{n}$ 'a fait que renforcer l'incertitude et 30 Le 4 février 2003, la République fédérale de Yougoslavie devient la Communauté d'États 
les tensions à propos du statut du Kosovo et donc, aussi, des droits de propriété. Le protectorat international ajoute à cette confusion au lieu de l'atténuer. De son côté, le Monténégro possède comme on l'a dit ses propres lois de privatisation et son système monétaire - des mesures hier encouragées par les puissances européennes. Celles-ci craignent aujourd'hui l'effet domino d'une séparation officielle du Kosovo et du Monténégro sur les très fragiles États multinationaux de Macédoine et de Bosnie-Herzégovine.

Cependant, c'est la perte des fonctions et des politiques de cohésion sociale des États qui accroît le risque d'explosions prenant des formes nationalistes et xénophobes. Le repli sur les communautarismes supposés protecteurs est le produit d'une désintégration des liens sociaux aggravée par les privatisations généralisées. L'instabilité politico-sociale est, à son tour, une cause majeure de la faiblesse des IDE dans une région à risque comme les Balkans occidentaux ${ }^{31}$, singulièrement dans des protectorats tels le Kosovo ou la Bosnie, sans claire délimitation des États et de la propriété (Papic, 2001 ; Solioz \& Dizdarevic, 2001, 2003 ; Stojanov, 2001).

La construction européenne est-elle le moyen d'une recomposition stabilisatrice ? Elle pourrait l'être dans la mesure où la coexistence de communautés conflictuelles serait facilitée dans un cadre plus large qui relativiserait, en outre, le rôle des frontières. Mais il aurait alors fallu qu'elle fût à même d'accueillir l'ensemble des Balkans occidentaux et d'offrir un cadre de cohésion sociale et de coopération multinationale respectant l'égalité des droits.

Or la désagrégation des solidarités est déjà à l'œuvre dans les États membres de l'UE, ne serait-ce que dans une Lombardie refusant de financer l'Italie du Sud ou dans une Flandre réticente à l'idée de partager des fonds avec la Wallonie... Elle fragilise l'Union européenne quand l'élargissement à des États plus pauvres que la moyenne s'accompagne non pas d'une extension du budget de l'Union mais de son plafonnement, qui conduira peut-être, comme certains le craignent, à prendre au Sud pour donner un peu à l'Est et à réduire les aides allouées aux nouveaux membres par rapport à celles obtenues par les anciens. Le Pacte de stabilité des Balkans lancé en 1999 à la fin de l'intervention militaire de l'OTAN se voit, lui aussi, allouer très peu de ressources. Et il

de Serbie-et-Monténégro sur la base de l'accord de Belgrade du 14 mars 2002, signé sous la pression internationale, qui reportait de trois ans le droit à l'autodétermination.

31 C'est la terminologie utilisée par les institutions européennes pour qualifier les républiques de l'ancienne Yougoslavie (à l'exception de la Slovénie), la Roumanie et la Bulgarie (dont l'adhésion à l'UE est repoussée à 2007) et l'Albanie. 
apparaît, non comme une antichambre de l'Union, mais comme la périphérie d'une construction où la coordination des polices contre une criminalité montante remplacera une véritable politique de cohésion sociale. Comment celle-ci pourrait-elle être mise en œuvre alors que la baisse des impôts sur le capital et la remise en cause de la protection sociale sont privilégiées afin d'attirer les capitaux privés ?

Le sommet européen de Thessalonique en juin 2003 a réitéré l'objectif de stabilisation et d'association des Balkans occidentaux à la construction européenne. Cet engagement restera un vœux pieux tant que les mêmes politiques continueront à produire les mêmes effets déstabilisants, autrement dit, tant que l'on ne dressera pas le bilan des transformations systémiques (la transition) qui conditionnent l'intégration dans l'Union européenne. Ce bilan sera aussi celui du modèle social européen et permettra d'éclairer les causes des interrogations (et de la désaffection) croissantes à son égard.

\section{BIBLIOGRAPHIE}

Andreani T., dir. (2003), Le socialisme de marché à la croisée des chemins, Pantin: Le temps des cerises.

AndrefF W. (1993), La crise des économies socialistes, Grenoble : PUG.

ANDREFF W. (1999), "Privatisation et gouvernement d'entreprise dans les économies en transition : une évaluation critique”, in A. Gawerski, éd., op.cit., pp. 21-56.

ANDREFF W. (2001), "L'investissement direct étranger dans le développement inégal des pays en transition", Nouveaux Cahiers de l'IUED, Genève, Paris : PUF, pp. 127-152.

ANDRefF W., éd. (2002a), Analyses économiques de la transition postsocialiste, Paris : La Découverte, coll. Recherches.

AnDREFF W. (2002b), "Le pluralisme des analyses économiques de la transition", in W. Andreff (2002a), pp. 269-334.

Banque mondiale (2002), Transition. Les dix premières années.

Bатакоvic D. T. (1993), Kosovo, la spirale de la haine, Lausanne : L'Âge d'homme.

B ARTLETT W. (1999), "La transformation économique et la politique de développement des petites entreprises dans les États successeurs de la Yougoslavie", in RECEO (1999b), pp. 15-34.

B AFOIL F. (2002), Après le communisme, Paris : Armand Colin.

Betтelheim C. (1968), La transition vers l'économie socialiste, Paris : Maspero.

Betтelheim C. (1970), Calcul économique et formes de propriété, Paris : Maspero.

Blanchard O. (1997), The Economics of Post-Communist Transition, Oxford: Clarendon.

Bornstein M. (1997), "Non standard methods in the privatisation strategies of the Czech Republic, Hungary and Poland", Economics of transition, 5 (2), pp. 323-338.

Bougarel X. (1996), Bosnie. Anatomie d'un conflit, Paris : La Découverte.

Braudel F. (1985), La dynamique du capitalisme, Paris : Arthaud. 
Brus W. (1968), Problèmes généraux du fonctionnement de l'économie socialiste, Paris : Maspero.

CDC IXIS (2002), Flash Marché émergents, Restructuration des systèmes bancaires de l'Est, 5 février, n ${ }^{\circ} 2002-04$.

CERI - Études (1999-2001) (dir. J.-P. Pagé), Tableau de bord des pays de l'Europe centrale et orientale, Paris : Fondation nationale des sciences politiques.

Chavance B. (1994), La fin des systèmes socialistes, Paris : l'Harmattan.

Chavance B. (1996), "Évolution et extinction des systèmes socialistes", in IRM, op.cit., pp. 57-62.

Chavance B. (1999), "Le capitalisme et le socialisme comme espèces systémiques: formation, co-évolution, transformation", in B. Chavance et al., éds., op.cit., pp. 295-316.

Chavance B., Magnin E., Motamed-Nejad R. \& Sapir J., éds. (1999), Capitalisme et socialisme en perspective : évolution et transformation des systèmes économiques, Paris : La Découverte.

Clément K., éd. (1999), Que sont les espoirs devenus ? Dix ans après la chute du Mur, mythes et réalités des "transitions” à l'Est, Paris : Mouvements $\mathrm{n}^{\circ}$ 6, nov.-déc.

CléMENT K. (2000), Les ouvriers russes dans la tourmente du marché, Paris : Syllepse.

Coriat B. \& Weinstein O. (1995), Les nouvelles théories de l'entreprise, Paris : Le livre de poche.

Cuckovic N. (1997), "The grey economy and the privatisation process in Croatia", Reform Round Table Document $n^{\circ} 18$, Zagreb: IMO.

Derens J.-A \& Samary C. (2000), Les conflits yougoslaves de A à Z, Paris : Éd. de l'Atelier.

Despiney-Zochowska B. \& Samary C., éds. (1992), État, propriété et rapports sociaux en transition, Paris : IMSECO-CNRS.

Dullas M. (1962), La nouvelle classe dirigeante, Paris : Plon.

Dislas M. (1979), Une guerre dans la guerre. La révolution de Tito 1941-1945, Paris : Robert Laffont.

Drweski B. (2002), 'Du Parti 'ouvrier' à la 'gauche démocratique'. Les métamorphoses d'un parti de pouvoir polonais (1989-2001)", in J.-M. de Waele, éd., Partis politiques et démocratie en Europe centrale et orientale, Université de Bruxelles, coll. "Sociologie politique", pp. 71-83.

DuchÊNE G. \& Rusin P. (2002), "Les micro-entreprises innovantes dans la transition : le cas de la Pologne", in W. Andreff (2002a), éd., op.cit., pp. 19-60.

Eyal G ., Szelenyi I. \& Townsley E. (1998), Making Capitalism without Capitalists - The New Ruling Elites in Eastern Europe, London, New York: Verso.

Favarel-Garrigues G., dir. (2003), Criminalité, police et gouvernement : trajectoires postcommunistes, Paris : l'Harmattan, coll. Logiques politiques.

FéLICE M. DE (1995), "Slovénie des atouts mis à profit”, in E. Lhomel, op.cit., pp. 209-217.

Gabrie H. \& JACquiER J.-L. (1994), La théorie moderne de l'entreprise, Paris : Economica.

Garde P. (2000), Vie et mort de la Yougoslavie, (nouvelle édition), Paris : Fayard.

Garton Ash T. (1993), We the People, London: U.K. Penguin Book.

Gawerski A., éd. (1999), Les changements dans le secteur public : acteurs, dilemmes et résultats (expériences de la France et de la Pologne), Warszawa : Szkola Glowna Handlowa (Éd. franco-polonaise).

Glamoсак M. (2002), La transition guerrière yougoslave, Paris : l'Harmattan.

Goati V., Ed. (1995), Challenges of Parliamentarism: The case of Serbia in the early nineties, Belgrade: Institute of Social Sciences. 
Gorzelak G., Ehrlich E., Faltan L. \& Illner M., Eds. (2001), Central Europe in Transition: Towards EU Membership, Regional Studies Association, Polish Section, Warsaw: Scholar.

G17 Plus (1999), Final account - economic consequences of NATO bombing: estimate of the damage and finances required for the economic reconstruction of Yugoslavia, Belgrade: Dinkic M. Ed., Stubovi Kulture.

Horvat B. (1981), "Autogestion, efficacité et théorie néo-classique", in A. Dumas, éd., L'Autogestion, un système économique ?, Paris : Dunod, pp. 229-236.

Horvat B. (1992), "L'économie yougoslave - les caprices de l'économie", in M. Morokvasic, éd., op.cit., pp. 7-33.

IRM (1996), Relations sociales et acteurs sociaux à l'Est (Actes du colloque de l'Institut d'études marxistes), Paris : Espaces Marx/l'Harmattan.

Jarosz M., Ed. (2000), Ten Years of Direct Privatisation, Warszawa: Institute of Political studies.

Johnstone D. (2002), The Fool's Crusade, London: Pluto Press.

Jugoslovenski Pregled/Yugoslav Survey (1997, 2001), Privatizacija u Srbiji, Propisi $i$ primena/Privatisation in Serbia, Regulations and Applications, Belgrade.

KARDELj E. (1976), Les contradictions de la propriété sociale dans le système socialiste, Paris : Anthropos.

Kornai J. (1984), Socialisme et économie de la pénurie, Paris : Economica.

Kornai J. (1990), The Road to a Free economy - Shifting from a Socialist Country: The Example of Hungary, New York, London: W.W. Norton \& Company.

Kornai J. (1996), Le système socialiste. L'économie politique du communisme, Grenoble : Presse universitaires de Grenoble.

Kornai J. (2001), La transformation économique postsocialiste - dilemmes et décisions (édité par B. Chavance et M. Vahabi), Paris: Éditions de la Maison des Sciences de l'Homme.

Kubli O.-L. (1998), Du nationalisme yougoslave aux nationalismes post-yougoslaves, Paris : l'Harmattan.

Lazic M., Ed. (1995), Society in Crisis - Yugoslavia in the Early 90s, Belgrade: Filip Visnjic.

Lazic M., Ed. (1999), Protest in Belgrade, Budapest, New York: Central European University Press.

Lemoine F. (2003), L'économie chinoise, Paris : La Découverte, coll. Repères.

Lewin M. (1997), “Anatomie d'une crise”, in R. Motamed-Nejad, éd., op.cit., pp. 41-80.

Lewin M. (2003), Le siècle soviétique, Paris : Fayard/Le Monde diplomatique.

Lhomel E., éd. (1995), Transitions économiques à l'Est 1989-1995, Paris : La Documentation française.

Lhomel E., éd. (2000), “L'Europe centrale et orientale. Dix ans de transformations (1989-1999)", Les études de la Documentation française, CEDUCEE.

Magas B. (1993), The Destruction of Yugoslavia - Taking the Break-up 1980-1992, London, New York: Verso.

Mandel E. (1974), "Ten theses on the social and economic laws governing the society transitional between capitalism and socialism", Critique, A Journal of Soviet Studies and Socialist Theory, n³, Autumn, pp. 5-23.

Mandel E. (1977), "Sur la nature de l'URSS", Critique Communiste, numéro spécial “Qu'est-ce que l'URSS ?”, octobre.

Mandel E. (1991), Fallacies of State Capitalism, London: Socialist Outlook. 
Marx K. (1963), “Le Capital”, in CEuvres, Économie I, Paris : La Pléiade.

Marx K. (1968), "Principes d'une critique de l'économie politique", in Oeuvres, Économie II, Paris : La Pléiade.

Masson D. (2002), L'utilisation de la guerre dans la construction des systèmes politiques en Serbie et en Croatie, 1989-1995, Paris : l'Harmattan.

Matvejevitch P. (1996), Le monde des "ex" - confessions, Paris : Fayard.

Mink G. \& SzuReK J.-C. (1992), Cet étrange post-communisme : ruptures et transitions en Europe centrale et orientale, Paris : La Découverte.

Mink G. \& Szurek J.-C. (1999), La Grande conversion, le destin des communistes en Europe centrale, Paris : Le Seuil.

Menciger J. (2000), "Macroeconomic policies and achievements in transition economies, 1989-1999”, UNECE, Economic Survey of Europe, pp. 89-94.

Menciger J. (2002), "Foreign direct investment and convergence", Conference on Eastern Enlargement as an all European development project, (Manuscript), Budapest.

Morokvasic M., éd. (1992), "Yougoslavie : logiques de l'exclusion”, numéro spécial de Peuples méditerranéens, 61, octobre-décembre.

Motamed-Nejad R., éd. (1997a), URSS et Russie - rupture historique et continuité économique, Actuel Marx confrontation, Paris : PUF.

Motamed-NeJAd R. (1997b), "Salariat, rapports marchands et transformations en Russie", in R. Motamed-Nejad (1997a), pp. 221-258.

Motamed-Nejad R. (1999), "Approches de la transition et nature des économies postsocialistes", in RECEO (1999a), pp. 11-60.

NAGels J. (1991), Du socialisme perverti au capitalisme sauvage, Bruxelles : Éditions de l'Université de Bruxelles.

NAGELS J. (1996) (en collaboration avec R. Walch), "Les conditions préalables pour construire la première phase de la transition vers le marché en Russie : la phase du capitalisme sauvage", in IRM, op.cit., pp. 279-286.

Naville P. (1970), Le nouveau Léviathan - Le salaire socialiste, Paris : Anthropos.

Nellis J. (1999), “Time to rethink privatization in transition economies?", Transition, 10 (1), pp. 4-6.

Nouveaux cahiers de l'IUED (2001), Du socialisme à l'économie de marché-errances de la transition (édité par A. November), Paris : PUF, Coll. Enjeux.

OCDE (2002), Évaluation économique de la République fédérale de Yougoslavie (site OCDE).

PAPIC Z., Ed. (2001), International Support Policies to Southeast European Countries: Lessons (not) Learned in B-H, Sarajevo: Müller.

Polany K. (1957), The Great Transformation: The Political and Economic Origins of Our Times, New York: Rinehart.

Popov N., éd. (1998), Radiographie d'un nationalisme - Les racines serbes du conflit yougoslave, Paris : Éd. de l'Atelier.

Privatizacija u Srbiji/Privatisation in Serbia (1997, 2001), Jugoslovenski Pregled/Yugoslav Survey.

RECEO - Revue d'études comparatives Est-Ouest (1999a), Les économies post-socialistes : une décennie de transformations, numéro spécial coordonné par E. Magnin \& R. MotamedNejad, vol. 30, $\mathrm{n}^{\circ}$ 2-3, juin-septembre, Paris : CNRS.

RECEO (1999b), Les transformations économiques dans la péninsule des Balkans, numéro spécial coordonné par Y. Rizopoulos, vol. 30, n 4, décembre, Paris : CNRS.

Rizopoulos Y. (1999), “Quelle est la spécificité des transitions balkaniques ?”, in RECEO 
(1999b), pp. 5-14.

Roland G. (1989), Économie politique du système soviétique, Paris : l'Harmattan.

Roux M. (1999), Le Kosovo. Dix clés pour comprendre, Paris : La Découverte.

RupNik J. (2001), "L'Europe du Centre-Est entre quête de stabilité et tentation populiste", in Études du CERI, n ${ }^{\circ}$ 81, décembre, pp. 15-18.

SAchs J. (1991), "Accelerating privatization in eastern Europe", World Bank Conference on Development Economics, Washington, D.C., 25-26 April.

SACHS J. \& Woo W.T. (1994), "Structural factors in the economic reforms of China, eastern Europe, and the former Soviet Union”, Economic Policy, 9 (18), pp. 102-145.

SAmARy C. (1988a), Le marché contre l'autogestion, l'expérience yougoslave, Paris : Publisud/La Brèche.

S Amary C. (1988b), Plan, marché et démocratie, l'expérience des pays dits socialistes, Cahiers d'étude et de recherche de l'Institut international de recherche et de formation, Amsterdam : Ed. IIRF.

SAmary C. (1992), "Quelques questions sur un tournant historique", in B. Despiney \& C. Samary, éds., op.cit., pp. 41-45.

SAMARY C. (1994), La déchirure yougoslave, questions pour l'Europe, Paris : l'Harmattan.

SAmary C. (1996), "Bases sociales de la restauration capitaliste en Europe de l'Est", in IRM, op.cit., pp.29-40.

SAmARy C. (1999a), "Le démantèlement programmé d'une fédération", in La nouvelle guerre des Balkans. Manière de voir - Le Monde Diplomatique, $\mathrm{n}^{\circ} 45$, mai-juin, pp. 36-41.

SAMARY C. (1999b), "L'indétermination théorique des critères d'efficacité. Questions ouvertes de la 'transition vers des économies de marché'", in A. Gawerski, éd., op.cit., pp. 58-72.

SAmary C. (1999c), "Autodétermination : le cas yougoslave", Actes du colloque international " La justice et la guerre ", Dialogue, $\mathrm{n}^{\circ}$ 31-32, Paris, automne/hiver, pp. 21-28.

SAmARY C. (1999d), "Mandel et les problèmes de la transition au socialisme", in G. Achcar, éd., Le marxisme d'Ernest Mandel, Actuel Marx Confrontation, Paris : PUF, pp. 129-171.

SAmary C. (2002), "Des privatisations forcées à la démocratie imposée : quels critères d'adhésion à l'Union européenne ?", La Revue internationale et stratégique, automne, Paris : IRIS, PUF, pp. 29-37.

SAPIR J. (1998), Le krach russe, Paris : La Découverte

SAPIR J. (1999), “À l'épreuve des faits... Bilan théorique des politiques macroéconomiques mises en œuvre en Russie”, in RECEO (1999a), pp. 153-215.

SAPIR J. (2000), Les trous noirs de la science économique, Paris : Albin Michel.

SAPIR J. (2002), Les économistes contre la démocratie - Pouvoir, mondialisation et démocratie, Paris : Albin Michel.

SEN A. (1999), L'économie est une science morale, Paris, La Découverte/Poche.

SEN A. (2000), Un nouveau modèle économique - développement, justice, liberté. Paris, Ed. Odile Jacob.

SGARD J. (1995), "Entre droit et marché : les révolutions légalistes à l'Est", Esprit, mai, repris dans Problèmes économiques, $\mathrm{n}^{\circ} 2441$ ("De la nécessité du droit et de l'État dans le passage à l'économie de marché"), 11 octobre, pp. 4-11.

SLIM A. (1996), "Quel capitalisme à l'Est? Le cas de la Bulgarie", in IRM, op.cit., pp. 41-61.

SLIM A. (1999), "Les trajectoires différenciées de la transition économique dans les Balkans", in RECEO (1999a), pp. 237-258.

Solioz C. \& Dizdarevic S.-A., Eds. (2001), Ownership Process in Bosnia and Herzegovina, 
Sarajevo. Édité par les auteurs avec le soutien de la Karl Popper Foundation, Zug, Suisse.

Solioz C. \& Dizdarevic S.-A., éds. (2003), La Bosnie-Herzégovine : enjeux de la transition, Paris : l'Harmattan.

Stark H. (1993), Les Balkans, le retour de la guerre en Europe, Paris : IFRI, Dunod.

Stiglitz J. (1999), Wither reform? Ten years of transition, Keynote address to the Annual World Bank Conference on Development Economics, Washington, D.C.: The World Bank.

Stiglitz J. (2002), La grande désillusion, Paris : Fayard.

Stojanov D. (2001), "Bosnia-Herzegovina since 1995: Transition and reconstruction of the economy”, in Z. Papic, Ed., op.cit., pp. 61-86.

SupeK R., éd. (1973), Bilan critique du socialisme yougoslave, Paris : Anthropos.

Szalai E. (1999), Post-socialism and Globalization, Budapest: Istvan Nemeth.

The Laws on enterprises and privatisation (1998, 2001), Belgrade: Yugoslav Chamber of Commerce and Industry.

Vukadinovic N. (1999, 2000, 2001), "Slovénie", Études du CERI, Paris : Fondation nationale des sciences politiques.

Walsh P.-P. \& Whelan C. (2001), "Firm Performance and Political Economy of Corporate Governance Survey. Evidence from Bulgaria, Hungary, Slovakia and Slovenia" Economic Systems, Vol. 25/2, June, pp. 85-112.

Woodward S.L. (1995), Balkan Tragedy - Chaos and Dissolution after the Cold War, Washington, D.C.: The Brookings Institution.

Zlotowsкi Y. (1998), La crise des paiements en Russie, expression d'un consensus social ?, Études du CERI, $\mathrm{n}^{\circ} 43$, août, Paris : Fondation nationale des sciences politiques. 